\title{
Effect of interface fields on the piezoelectric response of aluminum nitride thin films
}

John P. Harman

West Virginia University

Follow this and additional works at: https://researchrepository.wvu.edu/etd

\section{Recommended Citation}

Harman, John P., "Effect of interface fields on the piezoelectric response of aluminum nitride thin films" (2008). Graduate Theses, Dissertations, and Problem Reports. 2669.

https://researchrepository.wvu.edu/etd/2669

This Thesis is protected by copyright and/or related rights. It has been brought to you by the The Research Repository @ WVU with permission from the rights-holder(s). You are free to use this Thesis in any way that is permitted by the copyright and related rights legislation that applies to your use. For other uses you must obtain permission from the rights-holder(s) directly, unless additional rights are indicated by a Creative Commons license in the record and/ or on the work itself. This Thesis has been accepted for inclusion in WVU Graduate Theses, Dissertations, and Problem Reports collection by an authorized administrator of The Research Repository @ WVU. For more information, please contact researchrepository@mail.wvu.edu. 
EFFECT OF INTERFACE FIELDS ON THE PIEZOELECTRIC RESPONSE OF ALUMINUM NITRIDE THIN

FILMS

by

John P. Harman

Thesis submitted to the College of Engineering and Mineral Resources

at West Virginia University

in partial fulfillment of the requirements

for the degree of

Master of Science

in

Electrical Engineering

Approved by

Dimitris Korakakis Ph.D., Committee Chairperson

Parviz Famouri Ph.D.

Xian-An Cao Ph.D

Lane Department of Computer Science and Electrical Engineering

Morgantown, West Virginia

2008

Keywords: Aluminum Nitride, Piezoelectric Response, Tensor, $d_{33}$, Thin Film, Electric Field, Interface Field

Copyright 2008 John P. Harman 


\section{Abstract}

\section{Effect of Interface Fields on the Piezoelectric Response of Aluminum}

\section{Nitride Thin Films}

\section{by John P. Harman}

Group III-Nitride wide bandgap semiconductors have attracted much attention in the optoelectronic and electronic research areas recently. III-Nitride semiconductor materials are attractive materials for use in optoelectronic and high speed electronics devices because they are direct bandgap semiconductors and the bandgap can be varied over a wide range. It has also been shown that the III-Nitride group of materials function exceptionally well in harsh environments. The piezoelectric properties of the III-Nitride material system have been studied and several of the III-Nitride compounds have been found to have non zero piezoelectric coefficients. This work shows that the observed piezoelectric coefficient of Aluminum Nitride (AIN) is directly related to the metal used as the topside contact. The data and preliminary analysis presented here indicate that AlN cannot be treated as an insulating material and must be treated as a semiconductor in order to model its piezoelectric behavior. 


\section{DEDICATION}

The author wishes to this work to my parents John and Honey Harman who made my college career possible. 


\section{ACKNOWLEDGMENTS}

I would like to express my deepest gratitude to my faculty advisor, Dr. Dimitris Korakakis, for his guidance, encouragement and funding throughout my undergraduate and graduate career. I would also like to thank my committee members Dr. Parviz Famouri and Dr. Andrew Cao for the untold hours they worked supporting this project. I would like to thank Dr. Kolin Brown for his guidance and training using the cleanroom equipment. Last but not least I would like to thank Vincent Pagan, Adam Kabulski, Rich Farrell, Kalyan Reddy Kasarla and Lee Rodak and the other members of our lab for their support of the project. 


\section{TABLE OF CONTENTS}

Abstract

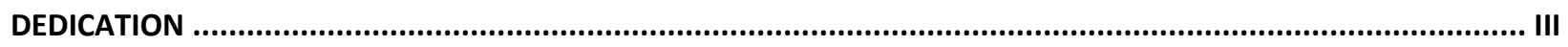

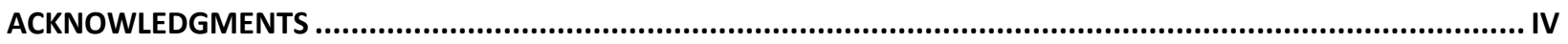

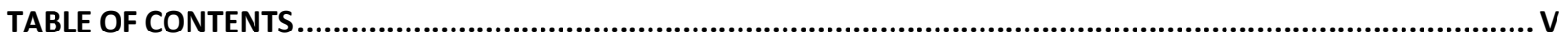

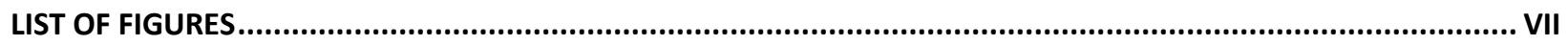

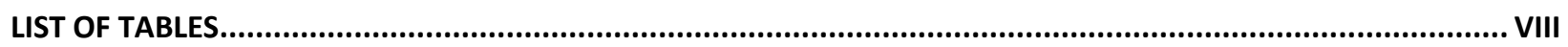

LIST OF EQUATIONS

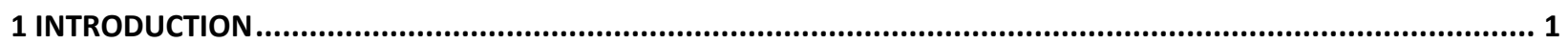

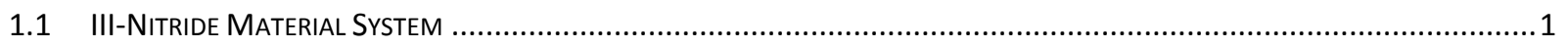

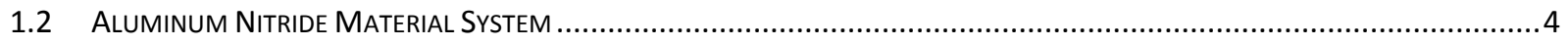

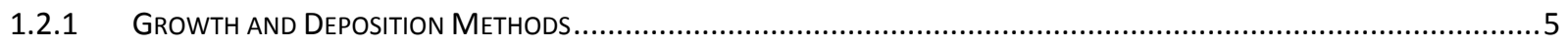

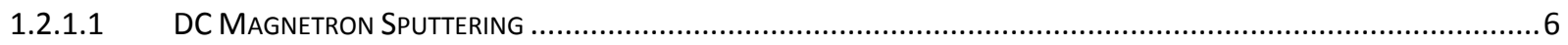

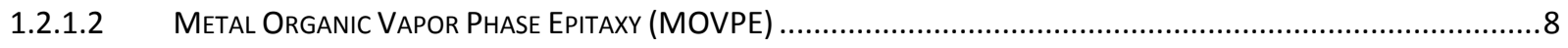

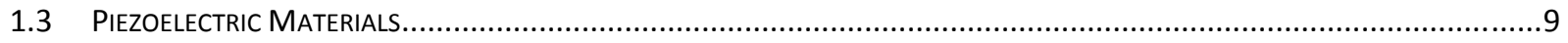

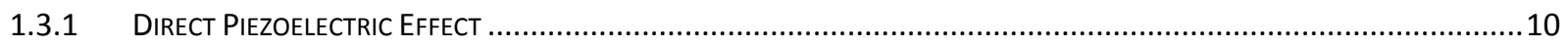

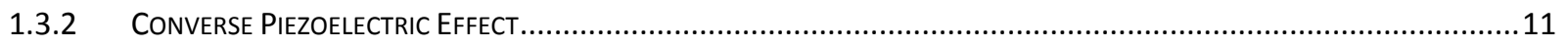

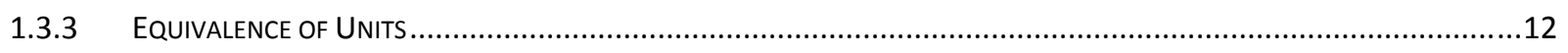

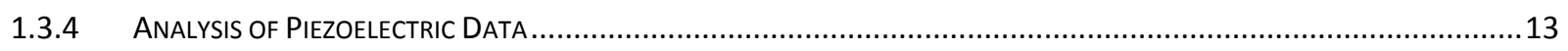

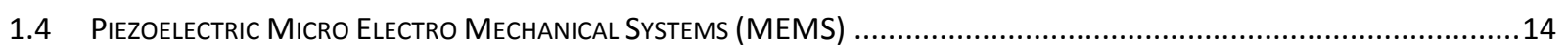

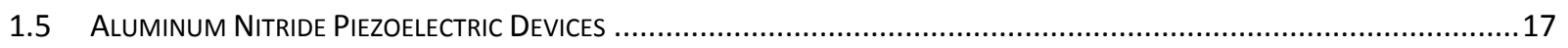

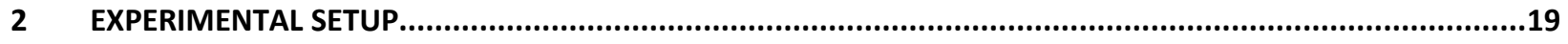

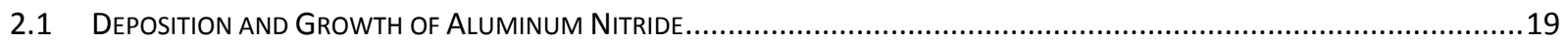




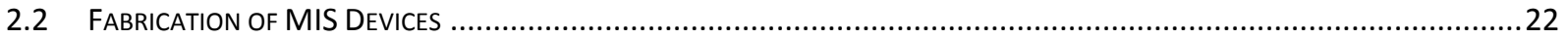

2.3 Determining the Piezoelectric Coefficient of Aluminum Nitride With LDV ...............................................24

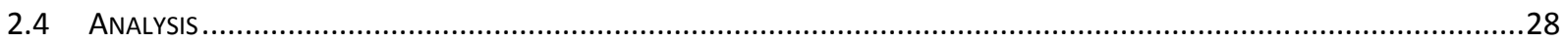

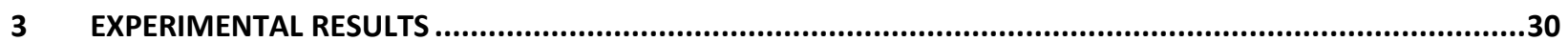

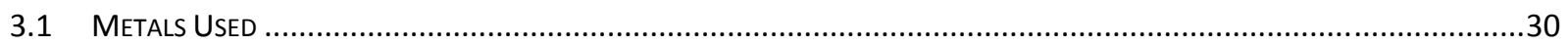

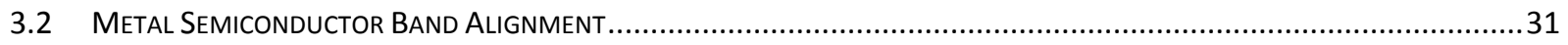

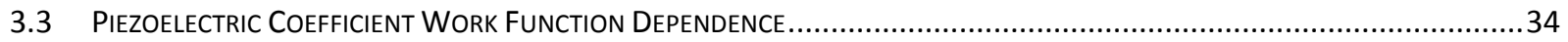

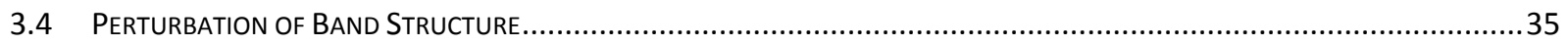

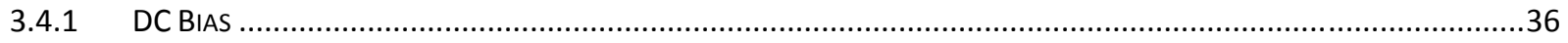

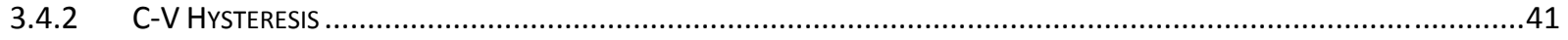

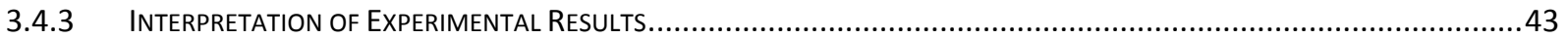

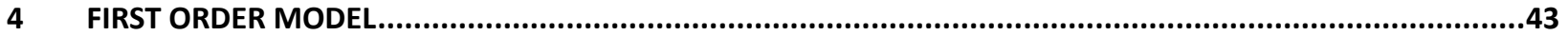

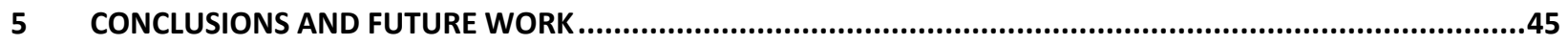

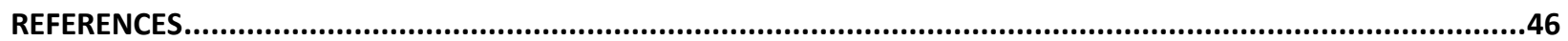

APPENDIX A - DEPOSITION OF ALUMINUM NITRIDE BY REACTIVE DC MAGNETRON SPUTTERING....................49

APPENDIX B - FABRICATION OF METAL INSULATOR SEMICONDUCTOR STRUCTURES .................................51

APPENDIX C - PROTOCOL FOR MEASURING $D_{33}$ BY LASER DOPPLER VIBROMETRY .....................................53

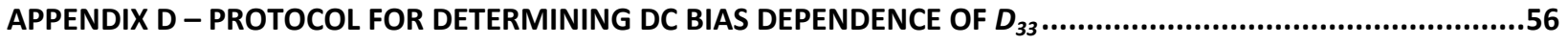




\section{$\underline{\text { LIST OF FIGURES }}$}

Figure 1 - III-Nitride Binary Compounds [13]......................................................................... 3

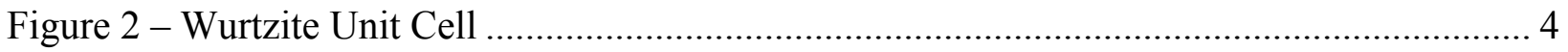

Figure 3 - Schematic representation of sputter deposition chamber............................................ 7

Figure 4 - Measurement of the Direct Piezoelectric Effect ............................................................ 11

Figure 5 - Schematic depiction of dual beam interferometer.................................................. 12

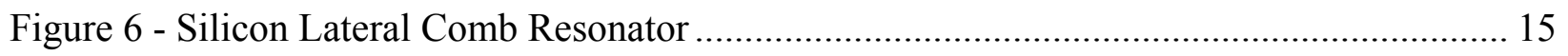

Figure 7 - Aluminum Nitride Thin Film Based MEMS Device …………………………........... 16

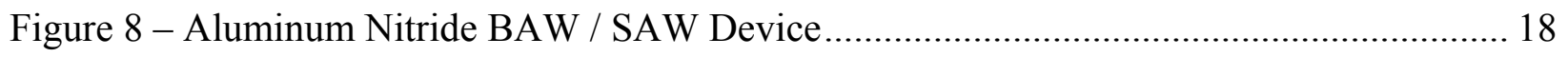

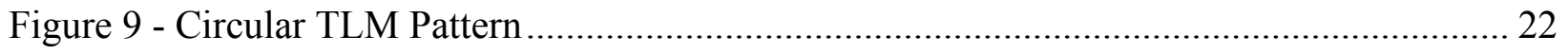

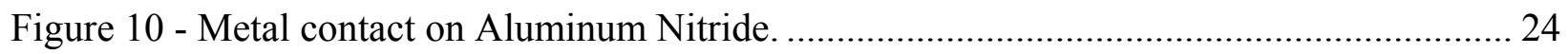

Figure 11 - Fringing Fields on Aluminum Nitride MIS Structure ……………………............ 25

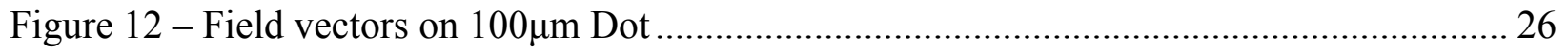

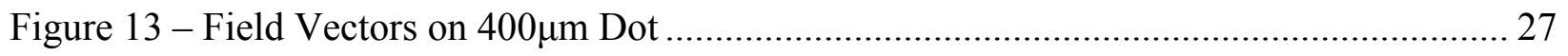

Figure 14 - Typical graph of displacement vs. voltage ............................................................ 29

Figure 15 - Band alignment between p-silicon and undoped Aluminum Nitride ........................ 33

Figure 16 - Maximum Electric Field Across Intrinsic Aluminum Nitride....................................33

Figure 17 - Piezoelectric Coefficient Dependence on Work Function ........................................ 34

Figure $18-d_{33}$ dependence on DC bias of DC reactive sputtered sample................................... 37

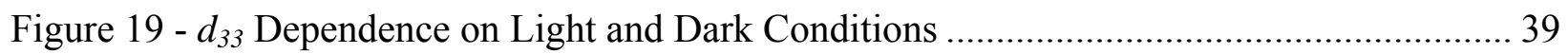

Figure 20 - MOVPE Aluminum Nitride Light and Dark............................................................. 40

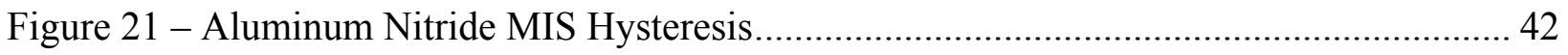




\section{LIST OF TABLES}

Table 1 - Common Aluminum Nitride Substrates ............................................................. 5

Table 2- Semiconducting Properties of Silicon Substrates ................................................ 19 


\section{LIST OF EQUATIONS}

Equation 1 - Wavelength and Bandgap Relation ....................................................................... 1

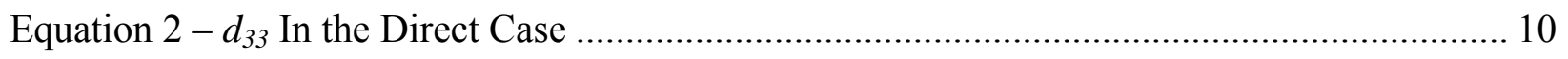

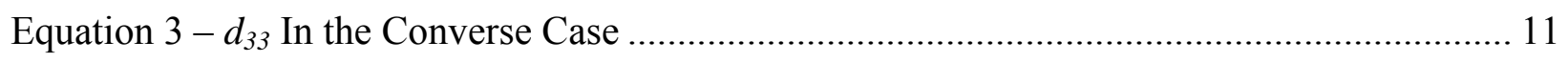

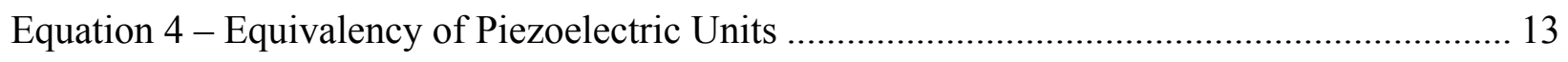

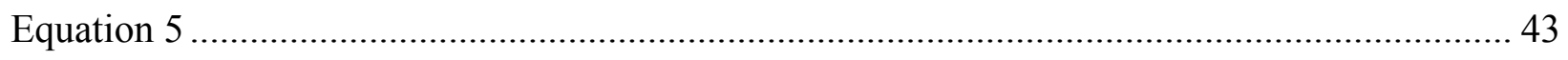

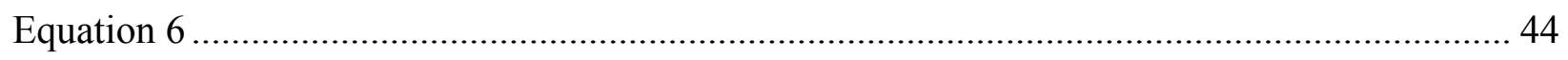

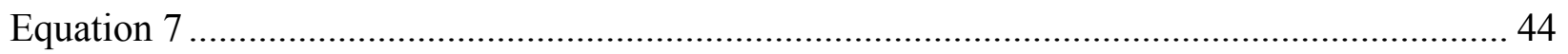

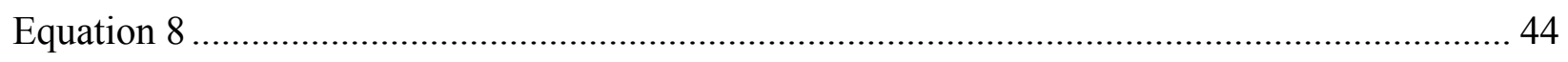

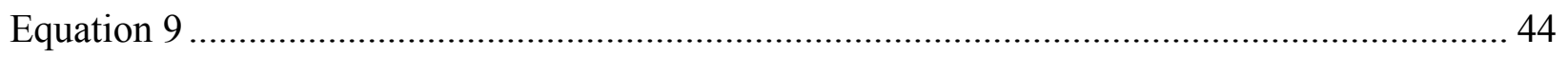

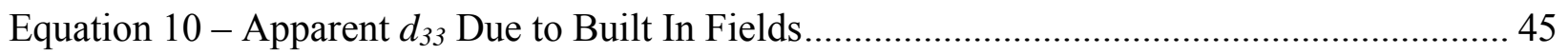




\section{INTRODUCTION}

\subsection{III-Nitride Material System}

The group III - Nitride material system consisting of the three binary compounds, namely Aluminum Nitride, Galium Nitride and Indium Nitride is of extreme interest to researchers in the optoelectronics, high power, high speed and high temperature electronics areas. [1] This interest is fueled largely because of the ability to tailor the bandgap of these semiconductor materials by varying the concentration of the constituent elements. Because the binary, ternary and quaternary compounds have similar lattice constants, it is possible to epitaxially grow homojunction structures that have minimal strain and defects. [2] By reducing strain and defects electronic and optoelectronic devices fabricated by hetero-epitaxial growth have higher efficiencies, faster switching speeds and can dissipate more heat than similar homojunction devices. III - Nitrides are inherently direct bandgap semiconductors. Direct bandgap semiconductors allow electrons to transition from the conduction band to the valence band without a change in momentum. [3] During this transition the electrons extra energy is expended in the form of a photon with wavelength proportional to the bandgap. The wavelength as a function of bandgap is given by Equation 1. [4]

$$
\lambda_{n m}=\frac{1240}{E_{g}(\mathrm{eV})}
$$

\section{Equation 1 - Wavelength and Bandgap Relation}

Equation 1 shows that the three binary III - Nitride compounds Indium Nitride (InN), Gallium Nitride (GaN) and Aluminum Nitride (AlN) will emit at 688, 400 and 200 nanometers respectively. This is also evidenced in Figure 1. By producing pseudobinary Aluminum Gallium Nitride $\left(\mathrm{Al}_{\mathrm{x}} \mathrm{Ga}_{1-\mathrm{x}} \mathrm{N}\right)$ and Aluminum Gallium Indium Nitride $\left(\mathrm{Al}_{\mathrm{x}} \mathrm{Ga}_{\mathrm{y}} \mathrm{In}_{1-\mathrm{x}-\mathrm{y}} \mathrm{N}\right)$ ternary and 
quaternary alloys it is possible to vary the bandgap almost continuously from that of InN all the way to AlN. Both emitting and detecting devices can be fabricated for specific wavelengths using this material system.

III - Nitride materials also exhibit piezoelectric properties that stem from the inherent dipole moment of the material system. The three aforementioned binary compounds each exhibit strong c-axis piezoelectric responses. While other III-Nitride ternary and quaternary compounds do exhibit piezoelectric properties, the magnitude of the response is much less compared to that of the binary compounds. [5] The piezoelectric response of the III - Nitride material system is of great interest because the material can simultaneously perform optoelectronic, electronic and piezoelectric functions. The piezoelectric coefficient of the materials in the III - Nitride system is also not degraded by high temperature or most harsh chemical environments. [5] Because of this thermal and chemical resistance the III - Nitride system lends itself nicely to sensors and actuators that must function in harsh environments.

Because of growth constraints, most III - Nitride materials are grown as thin films on a surrogate substrate such as Sapphire or Silicon. III - Nitride compounds can be synthesized using standard semiconductor growth techniques such as Metal Organic Vapor Phase Epitaxy (MOVPE) [6], Molecular Beam Epitaxy (MBE) [7] and reactive sputtering [8], [9], [10]. Generally, MOVPE and MBE produce very high quality single crystal films whereas reactive sputtering produces polycrystalline or amorphous films. However, MOVPE and MBE are inherently slow processes that do not lend themselves to high throughput as is required in the microelectronics industry. Reactive sputtering can achieve the high throughput necessary for a production environment at 
the cost of crystalline quality. Much research has been conducted with the goal of improving the crystalline quality of films deposited by reactive sputtering. Despite these efforts the current state of the art sputtered films are less efficient than MOVPE or MBE films. [11] Because sputtering is inherently a random process, sputtered films tend to be polycrystalline or amorphous as opposed to single crystals. [12] Therefore, when these films are employed in optoelectronic or electronic devices leakage currents tend to be higher, electronic and optical efficiency tends to be lower.

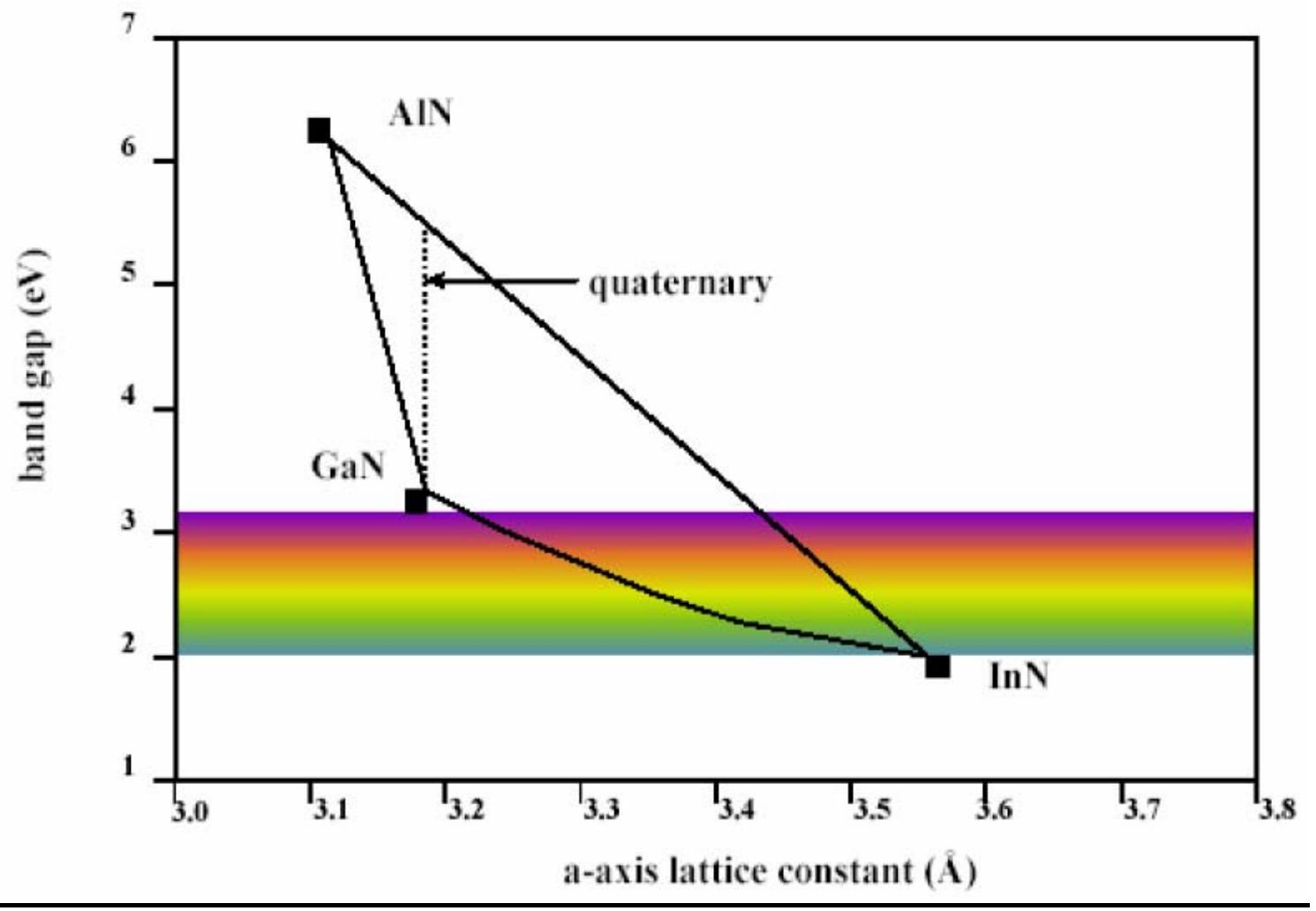

Figure 1 - III-Nitride Binary Compounds [13] 


\subsection{Aluminum Nitride Material System}

Aluminum Nitride (AlN) is III - Nitride and a wide bandgap semiconductor material that crystallizes in the wurtzite structure with lattice constant $\mathrm{a}=3.11 \AA$ and $\mathrm{c}=4.98 \AA$. [14] The wurtzite crystal structure is shown in Figure 2.

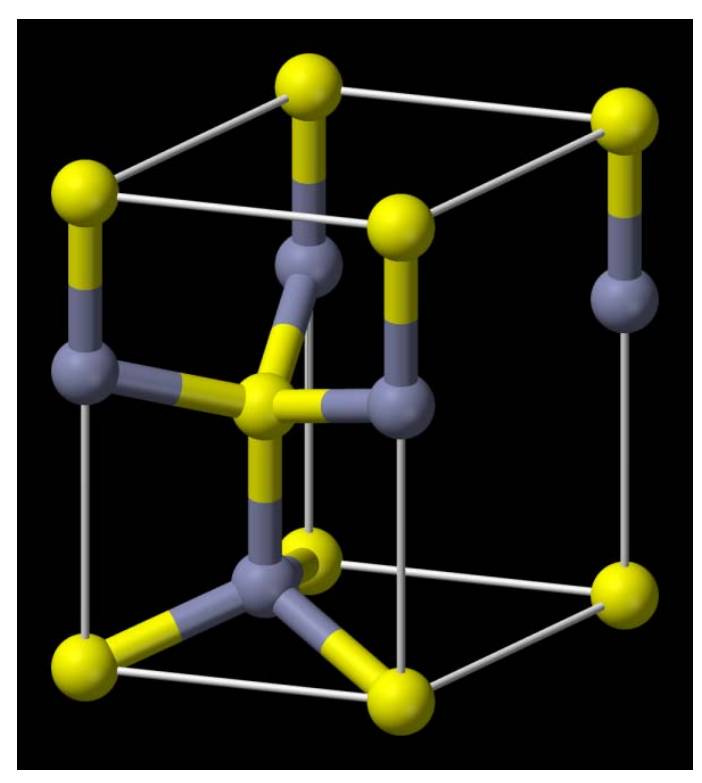

Figure 2 - Wurtzite Unit Cell

The bandgap of AlN is $6.22 \mathrm{eV}$ making it both a viable dielectric [15] and semiconductor [16] material. Single crystalline AlN is very hard to manufacture and most AlN samples are either amorphous or polycrystalline. The crystalline quality of AIN is directly related to the refractive index of the material. Generally, AlN has a refractive index between 1.8 and 2.1 with 1.8 representing amorphous AlN and 2.1 representing single crystalline AlN. [17] The Fermi level and impurity concentration of AlN is much harder to determine because standard photoluminescence and Hall Effect studies do not lend themselves to the measurement of wide bandgap semiconductors. The electron affinity of AlN is likewise not well known and some 
studies report that AlN actually has a negative electron affinity. Despite the difficulty in experimentally determining many of the electrical and semiconducting properties of AlN it is nonetheless a very important material in electronics and optoelectronics.

\subsubsection{Growth and Deposition Methods}

The high Gibbs free energy of Aluminum Nitride makes it challenging to synthesize high quality single crystalline Aluminum Nitride. Aluminum Nitride is generally not grown as a bulk material but rather as a thin film on a surrogate substrate with a significant lattice mismatch. The most common foreign substrates used to deposit Aluminum Nitride thin films and their lattice mismatch are summarized in Table 1.

\begin{tabular}{|c|c|}
\hline Material & Lattice Mismatch \\
\hline Silicon & $19 \%$ \\
\hline Aluminum & $8 \%$ \\
\hline Platinum & $11 \%$ \\
\hline
\end{tabular}

\section{Table 1 - Common Aluminum Nitride Substrates}

The use of Face Centered Cubic (FCC) metals to act as a substrate for the growth of Aluminum Nitride allows higher crystalline quality at the expense of creating a Schottky contact on the backside of the Aluminum Nitride thin film. In some electronic applications this is a fortuitous intersection of two desirable traits but in most applications the proximity of the conductor actually hinders the electrical performance of the device.

The two most prominent methods of reactively synthesizing Aluminum Nitride are reactive sputtering and Metal Organic Vapor Phase Epitaxy (MOVPE). 


\subsubsection{DC Magnetron Sputtering}

The synthesis of Aluminum Nitride by DC Magnetron reactive sputtering involves simultaneously populating a vacuum chamber with Nitrogen gas and atomic Aluminum. Sputtering is accomplished by evacuating a vacuum chamber to a base pressure of less than $10 \mathrm{e}-$ 5 Torr and introducing a mixture of Argon and Nitrogen gasses into the chamber. A strong magnetic field is created by passing a DC current through a magnet embedded in a block of Aluminum inside the chamber. The powerful negative DC voltage applied to the Aluminum target ionizes the gas creating plasma above the Aluminum. The kinetic energy of the positively charged Nitrogen and Argon ions slamming into the negatively charged Aluminum target causes atomic Aluminum particles to be "sputtered" from the target. The particles travel away from the target in a Gaussian distributed pattern and react with the Nitrogen gas in the chamber to form Aluminum Nitride. Aluminum Nitride is reactively formed when the Aluminum atoms are deposited on the substrate. The general sputtering process is schematically outlined in Figure 3. 


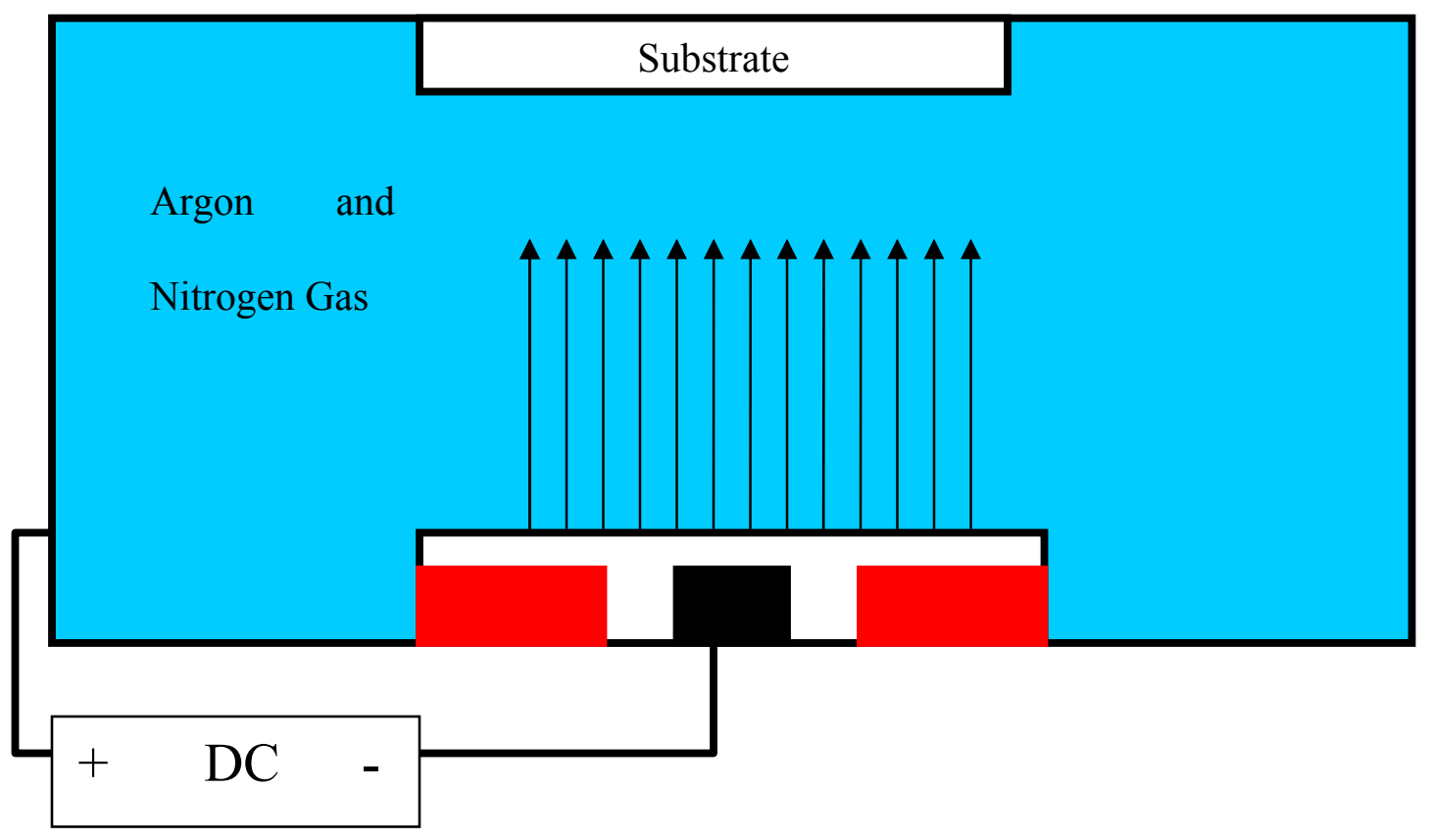

Figure 3 - Schematic representation of sputter deposition chamber.

Sputter deposition is an excellent way to quickly deposit Aluminum Nitride thin films on many substrates including Silicon, Saphire and metals. Despite the ease of deposition, Aluminum Nitride thin films deposited by DC reactive magnetron sputtering are inherently lower in quality than their counterparts that are grown by high temperature methods. Sputtered films tend to be amorphous or polycrystalline and have a refractive index between 1.8 and 1.95. Surface roughness of sputtered films is often observed to be as high as $50 \mathrm{~nm}$. The rate of sputter deposition is directly contingent on the pressure of the Argon and Nitrogen gas in the sputter chamber. Generally, higher Argon pressures correspond to higher deposition rates and lower quality films. However, at very low Argon pressures it is impossible to produce plasma thus limiting the Aluminum Nitride film quality. Deposition rates and film quality are also dependent on the ratio of Argon to Nitrogen. The final factor that influences deposition rate and film 
quality is the power that is applied to the Aluminum target. Generally, higher powers result in higher deposition rates but lower quality Aluminum Nitride films. To produce high quality films it is necessary to optimize each of these parameters for the particular sputter deposition chamber through an iterative process.

\subsubsection{Metal Organic Vapor Phase Epitaxy (MOVPE)}

MOVPE is the second technique employed in this study to deposit Aluminum Nitride. MOVPE generally produces highly c-axis oriented single crystalline Aluminum Nitride thin films with a minimum number of defects and dislocations. MOVPE Aluminum Nitride is deposited by flowing a metal organic gas, namely tri-methyl aluminum, over a substrate that is heated to $900^{\circ}$ to $1200^{\circ}$ Celsius. As the tri-methyl aluminum gas becomes hot the chemical bonds break down allowing the metal to drop onto the substrate and the constituent molecules to be extricated from the chamber. Nitrogen gas is introduced into the chamber at the same time and deposition of Aluminum Nitride on the substrate occurs.

MOVPE deposition rates are generally slower than sputter deposition rates but the films produced are much higher quality. MOVPE is not used to deposit AIN on metal substrates because of the risk of metal contaminating the chamber during the high temperature deposition. With MOVPE it is possible to intentionally add impurities to the Aluminum Nitride to shift the Fermi level making it n-type or p-type material. Despite the ability to add the aforementioned impurities it is very hard to determine the Fermi level post deposition and therefore all Aluminum Nitride used in this study was intrinsic. The refractive index of Aluminum Nitride

films deposited by MOVPE is generally between 1.95 and 2.05 indicating a high degree of crystallinity. MOVPE deposited samples routinely exhibit surface roughness less than $10 \mathrm{~nm}$ and 
it is not uncommon to measure surface roughness of MOVPE deposited Aluminum Nitride samples of less than $5 \mathrm{~nm}$.

\subsection{Piezoelectric Materials}

By definition a piezoelectric material is any solid material that produces a charge when stress is applied to the material. [18] Generally piezoelectric materials are crystalline materials that have a dipole structure. At equilibrium the separation between the atoms is such that the charge state is zero. However, when a strain force is applied to a piezoelectric material the dipole is forced closer together thus creating a non-zero charge. The piezoelectric response of a material is defined as the amount of charge produced per unit stress. Due to differing material configurations the magnitude of the piezoelectric response varies across a wide range. To fully understand the piezoelectric response it is necessary to delve into the tensor representation of the crystal lattice. [19] Despite the complex nature of tensors and tensor analysis it is fairly easy to understand the practical application of the tensor as it applies to the uniaxial piezoelectric response. The uniaxial piezoelectric response is defined simply as the charge produced across an axis when unit stress is applied along the same axis. Using an orthogonal coordinate system the piezoelectric response can be defined as the charge produced along axis $\mathrm{x}$ when unit stress is applied along axis $\mathrm{x}$. This is denoted as $d_{x x}$. [19] The uniaxial piezoelectric response ignores any charge that may be induced on the other axes. This approach lends itself well to most piezoelectric materials because generally the piezoelectric response along one axis is much greater than the response along the other axes. The realization that the piezoelectric response is much greater along one axis than the other two axes leads to two direct measurement techniques 
used to determine the magnitude of the piezoelectric response. Generally the piezoelectric response of greatest interest is $d_{33}$ or the charge produced per unit stress along the vertical axis.

\subsubsection{Direct Piezoelectric Effect}

The piezoelectric effect manifests itself in two measurable effects, namely the direct and converse piezoelectric effect. The direct effect is exactly as described in the preceding section; a strain is applied to a crystal lattice and a charge is produced along the axis that the strain was applied. The direct effect can be measured using a mechanical stress applied to the crystal in conjunction with a charge integrator. During application of mechanical stress the crystal produces charge which is gathered on the charge integrator and measured as a voltage. Because the voltage is directly proportional to charge it is possible to determine the charge produced by the crystal per unit stress. Therefore $d_{33}$ takes on the units of Coloumb per Newton. Because the magnitude of the response is very small the units of pico Coloumb per Newton are generally used.

$$
d_{33}=\frac{\text { Charge }}{\text { Force }}=\frac{\text { Coloumb }}{\text { Newton }}
$$

\section{Equation $2-d_{33}$ In the Direct Case}

Equation 2 shows the relationship between charge and force and the units of measure. A schematic diagram of a device to measure the direct piezoelectric effect is outlined in Figure 4. In Figure 4 a mechanical excitation is applied to a metal contact by means of a stylus or other rigid mechanical body. The excitation causes a charge to accumulate on the metal contacts on 
the top and bottom of the piezoelectric crystal. This charge is gathered on the charge integrator and read as a voltage.

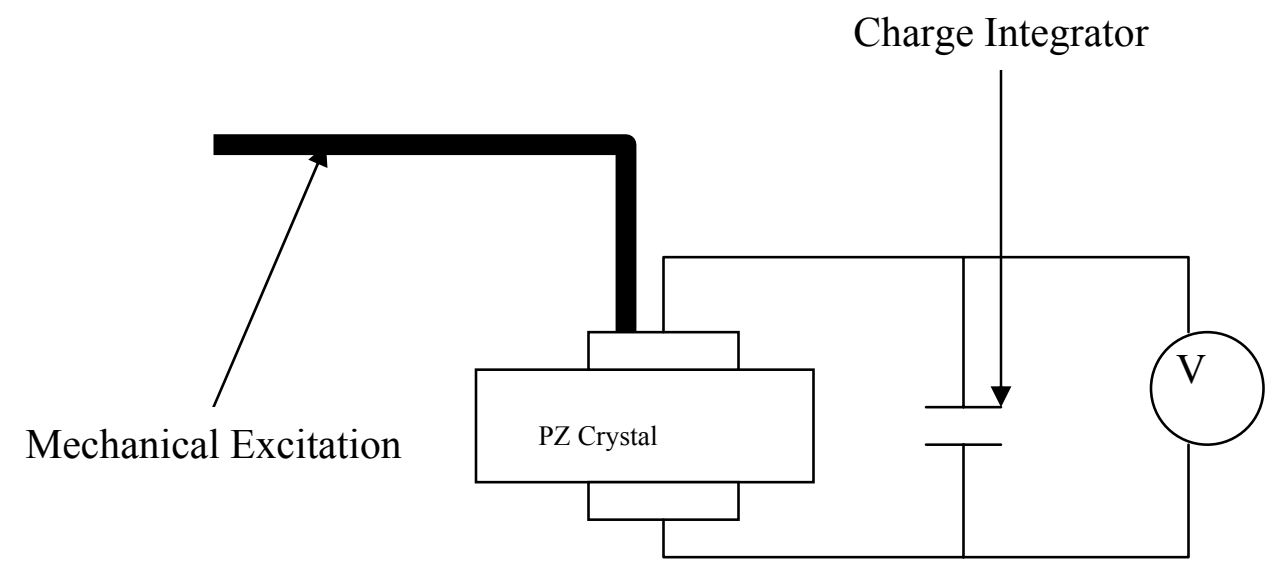

Figure 4 - Measurement of the Direct Piezoelectric Effect

\subsubsection{Converse Piezoelectric Effect}

The converse piezoelectric effect is functionalized by applying an electric field across the material and measuring the deformation of the crystal along the same axis that the field was applied. The piezoelectric coefficient, therefore, becomes displacement per unit field . In this case the units of $d_{33}$ are meters per volt or picometers per volt because of the very small displacements involved.

$$
d_{33}=\frac{\text { strain }}{\text { electric field }}=\frac{\text { displacement } / \text { thickness }}{\text { voltage } / \text { thickness }}=\frac{\text { displacement }}{\text { voltage }}=\frac{\text { picometer }}{\text { volt }}
$$

\section{Equation $3-d_{33}$ In the Converse Case}

Equation 3 shows the relationship between strain and electric field and shows that ideally $d_{33}$ does not depend on film thickness. The experimental setup for measuring the converse case 
generally involves depositing metallic contacts on opposite sides of the sample and applying an oscillating electric field between the top and bottom contacts. The oscillating electric field in turn produces an oscillating displacement along the axis. To measure this displacement many different methods have been employed. The most common methods involve the use of a dual beam differential interferometer or vibrometer to measure the picometer level displacements induced by the oscillating voltage. Other methods use extremely sensitive probe tips to physically probe the oscillating surface and measure the displacement. These methods, akin to Atomic Force Microscopy, are not often used because of their inherent complexity and low resolution. With optical methods it is possible to obtain resolutions of less than 5 picometers at frequencies as low as $1 \mathrm{kHz}$. A general dual beam interferometer / vibrometer setup is schematically depicted in Figure 5.

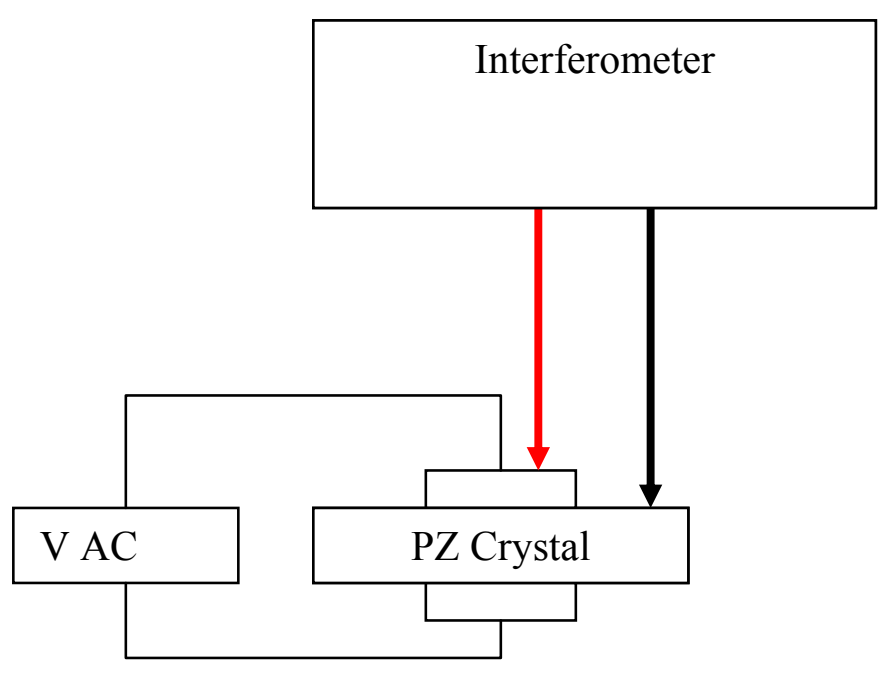

Figure 5 - Schematic depiction of dual beam interferometer

\subsubsection{Equivalence of Units}

It should be noted that the units of meters per volt and Coulomb per Newton are exactly equivalent. A simple unit conversion from meters per volt to Coulomb per Newton can be easily 
demonstrated. The unit meter is a fundamental unit and cannot be expressed in terms of simpler units. However the unit of volt can be expressed as a Joule per Coulomb allowing the following equation to be written:

$$
\frac{\text { meter }}{\text { volt }}=\frac{\text { meter }}{\frac{\text { Joule }}{\text { Coulomb }}}=\frac{\text { meter }}{\frac{\text { Newton }- \text { meter }}{\text { Coulomb }}}=\frac{\text { meter }- \text { Columb }}{\text { Newton }- \text { meter }}=\frac{\text { Columb }}{\text { Newton }}
$$

Equation 4 - Equivalency of Piezoelectric Units

From Equation 4 it is evident that the units in the direct case and the converse case are exactly equal.

\subsubsection{Analysis of Piezoelectric Data}

As with any experimental technique, the results obtained from direct measurement of the direct or converse piezoelectric effect are only as reliable as the analysis technique applied to the data. The analysis of charge versus stress or displacement versus voltage data can seem deceptively simple. However, to ensure that erroneous measurements are excluded it is imperative to validate all data by applying strict statistical analysis methods. The first step in analyzing any set of piezoelectric data is to perform a linear regression on the charge as a function of applied stress or the displacement as a function of applied voltage. Ideally the curve should be perfectly linear and pass through the origin. If the curve displays significant non-linearity i.e. the curve shows an exponential or polynomial dependence then the quality of the data immediately becomes questionable. Most piezoelectric response curves do not pass through the origin and have an intercept greater than the background noise or resolution of the measurement method. This phenomenon can not readily be explained and can generally be discounted. It is believed that this non-zero intercept is due to initial stress in the films due to defects and charge deformities. However, this is only a conjecture and has not been proven in this or other work. It is not 
prudent to force the regression through the origin because it can significantly change the piezoelectric coefficient. As with any analysis of experimental data it is imperative to determine the standard error of the measurements. The standard error is not simply the resolution of the measurement technique but rather the variability of the data set. As an example when measuring the converse piezoelectric response of an Aluminum Nitride thin film displacement data is taken for different values of excitation voltage. At each excitation voltage several (generally 100 or greater) single point measurements are averaged to produce the final measurement. The standard error generated by this data set is of extreme importance. To obtain an accurate estimate of the piezoelectric coefficient it is necessary to measure this standard error and use it when calculating the piezoelectric coefficient. The standard error is somewhat dependent on the signal to noise ratio (SNR) of the measurement technique. Therefore, data collected at lower applied stress levels or lower applied voltage levels generally exhibit a higher standard error. However, it should be noted that the standard error versus signal to noise ratio generally saturates at high SNR.

\subsection{Piezoelectric Micro Electro Mechanical Systems (MEMS)}

Recently, Micro Electro Mechanical Systems (MEMS) has come to the forefront of the microelectronics and micromechanics research field. MEMS have a wide variety of uses ranging from complex systems used as safe and arm devices for nuclear weapons to simple valves used to control the flow of minute amounts of fluids. MEMS are electromechanical systems that accomplish motion by one of two means either a static attraction repulsion as in silicon based MEMS or by means of a piezoelectric response as in Aluminum Nitride or other piezoelectric materials. Silicon based MEMS are by far the most prevalent and have been widely studied 
because of their compatibility with standardized silicon microchip processing technology. Figure 5 is a schematic representation of a lateral comb resonator (LCR) that is often employed to drive motion in Silicon based MEMS. In Figure 6 motion is along the $\mathrm{Y}$ axis.

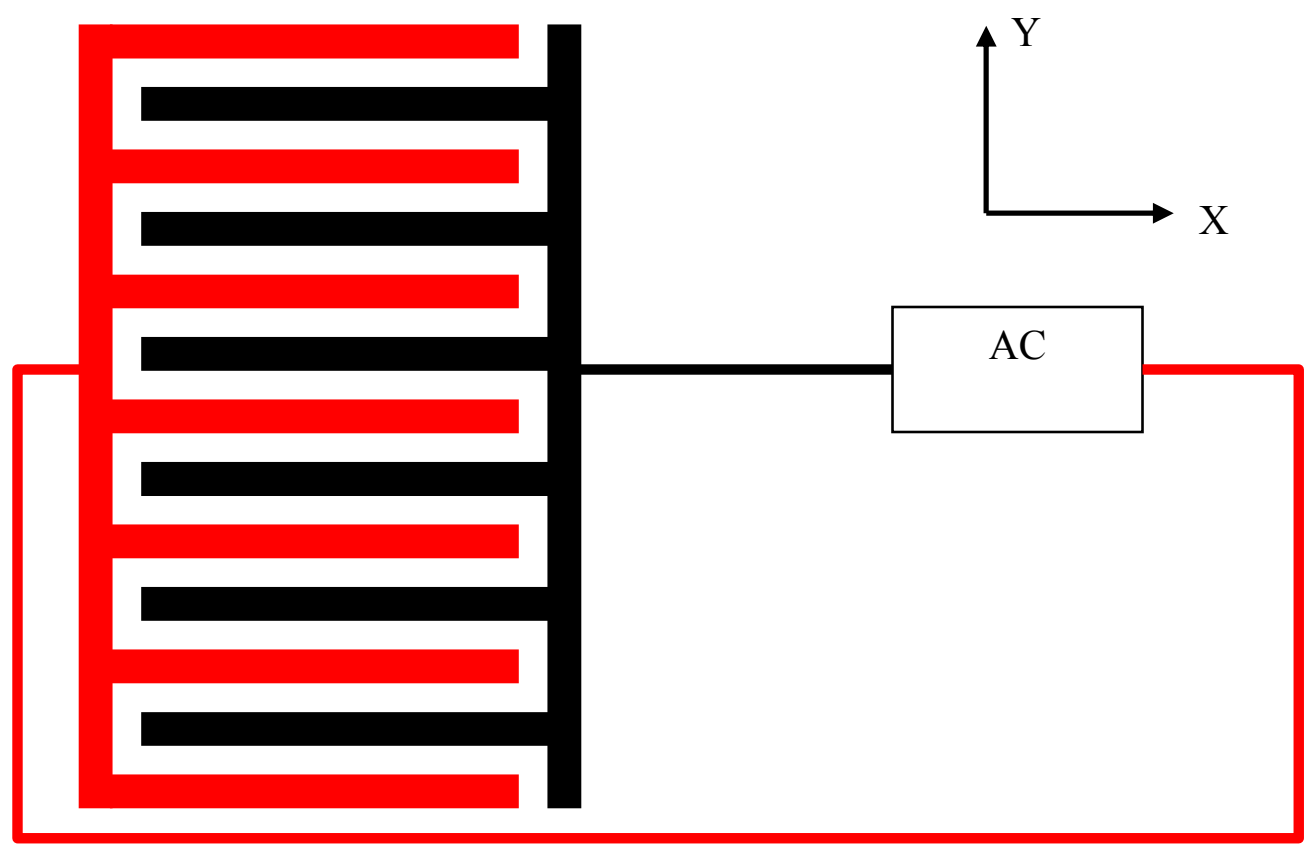

Figure 6 - Silicon Lateral Comb Resonator

Piezoelectric MEMS however, have only recently begun to be studied because the processing techniques that must be employed to fabricate complex structures from novel materials either do not exist or are very expensive and not well understood. The allure of using materials other than Silicon in MEMS lies in the fact that Silicon is greatly limited in the type of environment it can function in. High temperature and chemically harsh environments preclude Silicon based MEMS from being a viable solution. Lead Zircronate Titinate (PZT) is a very common piezoelectric material that is often employed in piezoelectric MEMS applications. PZT is an excellent candidate material for temperatures that do not exceed $200^{\circ} \mathrm{C}$. However, at high temperatures PZT loses its spontaneous polarization and the piezoelectric response rapidly 
declines. Aluminum Nitride maintains its piezoelectric properties at high temperatures and in harsh environments making it an excellent candidate material for piezoelectric MEMS applications that much withstand such environments. Because Aluminum Nitride is most easily deposited as a thin film, piezoelectric devices made using Aluminum Nitride generally incorporate a substrate material to aid in deposition. Figure 7 schematically depicts a simple cantilever MEMS device based on silicon and Aluminum Nitride thin film technology.

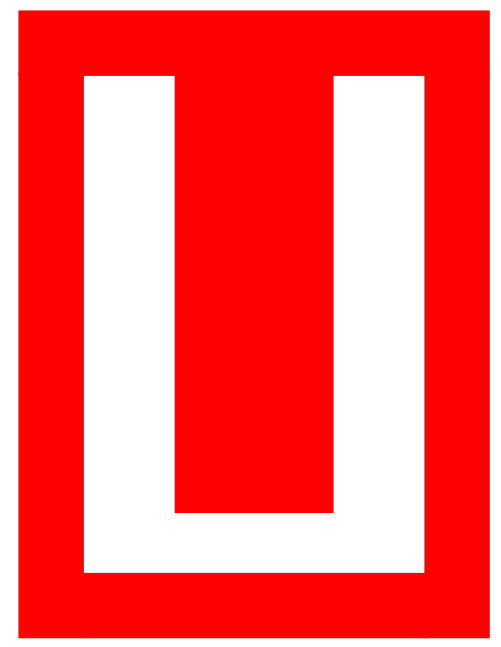

Top View

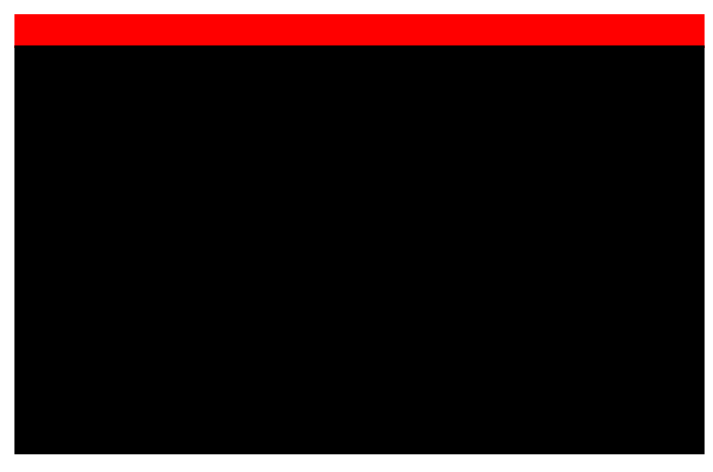

Side View

\section{Figure 7 - Aluminum Nitride Thin Film Based MEMS Device}

In Figure 7 red represents Aluminum Nitride, black represents silicon and white represents vacuum.

In Figure 7 a voltage is applied between the bottom of the structure (Silicon) and the top (Aluminum Nitride). The voltage and resulting electric field cause the Aluminum Nitride to actuate moving the cantilever either up or down depending on the polarity of the electric field. Conversely, if a stress is applied to the cantilever a charge will be produced that is proportional 
to the amount of stress applied. This utilization of both the direct and converse piezoelectric effect allows the cantilever MEMS device to act as both an actuator and a sensor. This dual functionality is a property that is unique to piezoelectric MEMS.

As with the deposition of Aluminum Nitride, fabrication techniques for Aluminum Nitride based piezoelectric MEMS present several challenges. Because of the chemical resistance of Aluminum Nitride it is not possible to use the same etching techniques that are utilized by the Silicon MEMS industry. Aqueous etching of Aluminum Nitride has proven to be very uncontrollable and does not lend itself to reliable MEMS releases. Dry etching techniques such as Reactive Ion Etching (RIE) and Deep Reactive Ion Etching (DRIE) have proven to be the most viable etching solutions for Aluminum Nitride MEMS. All chemical etching methods involve using a mask material to protect the areas of the Aluminum Nitride that must not be removed. Because Aluminum Nitride is very resistant to chemical etching few materials exist that can withstand the etching process, therefore, limiting the materials that can be used as a mask.

\subsection{Aluminum Nitride Piezoelectric Devices}

Aluminum Nitride as a candidate for piezoelectric MEMS systems was discussed in the previous section. However, other devices that utilize the piezoelectric properties of Aluminum Nitride also exist. These devices, while not new, have received much attention in the past few years. These devices fall into two major categories namely Bulk and Surface Acoustic Wave (BAW and SAW) devices and Metal Insulator Semiconductor (MIS) devices. Relative to Aluminum Nitride based piezoelectric MEMS these devices are much easier to fabricate. Generally the fabrication of BAW, SAW and MIS devices and structures involves depositing contact metal 
patterns using standard photolithographic techniques on an Aluminum Nitride substrate. Because complex etching techniques are not required to fabricate these devices it is much easier to mass produce them using mostly standard Silicon microchip processing techniques.

The first class of devices, BAW and SAW devices are of particular interest because they utilize both the direct and converse piezoelectric effect. Figure 8 is a schematic depiction of a BAW or SAW device.

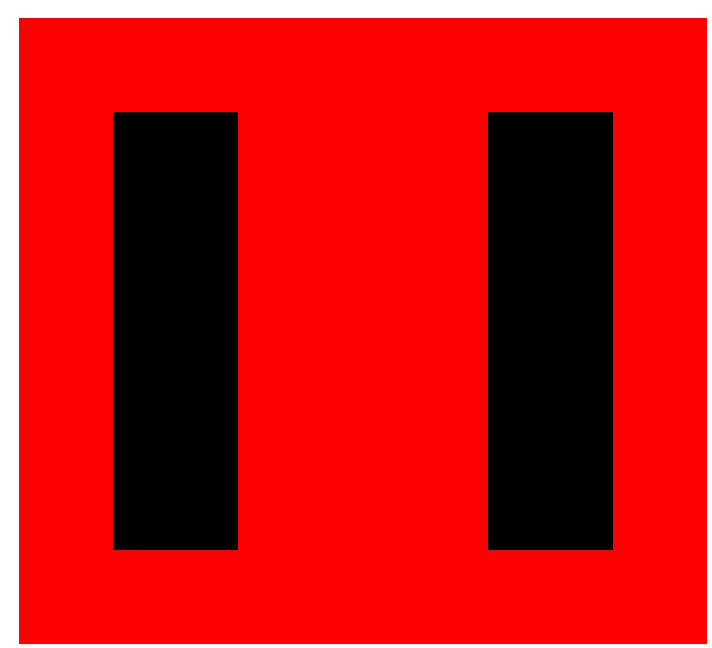

Top View

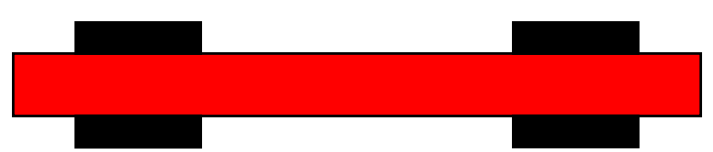

Side View

\section{Figure 8 - Aluminum Nitride BAW / SAW Device}

In Figure 8 red represents Aluminum Nitride while black represents metal contacts deposited on the Aluminum Nitride. The BAW / SAW device depicted in Figure 8 works by applying an oscillating voltage between the electrodes deposited on the left hand side of the device. This oscillating voltage induces movement in the Aluminum Nitride film. The movement causes acoustic waves to travel across the Aluminum Nitride to the opposing set of metal contacts. These contacts act as a transducer and a voltage can be measured across the contacts. Because the geometry of the device is easily controlled it is possible to tailor the frequency response of 
the device. The largest application of BAW / SAW devices is as a means of both electrical isolation and as a low pass, high pass or band pass filter.

\section{EXPERIMENTAL SETUP}

\subsection{Deposition and Growth of Aluminum Nitride}

All the Aluminum Nitride samples used in this work were deposited either by DC reactive magnetron sputtering or by Metal Organic Vapor Deposition (MOVPE). The protocol for depositing Aluminum Nitride by DC reactive magnetron sputtering is outlined in Appendix A and a general overview of DC reactive magnetron sputtering has been presented previously in this work.

The samples used in this work were deposited on three substrates, namely p-type silicon and n-type silicon. Table 2 outlines the properties of the silicon used in this study.

\begin{tabular}{|c|l|c|l|}
\hline & Resistivity & Bulk Carrier Concentration & Orientation \\
\hline n-type Si (Ph Doped) & $1-20 \Omega$-cm & $1.310^{15} \mathrm{~cm}^{-3}$ & $(100)$ \\
\hline p-type Si (B Doped) & $1-20 \Omega-\mathrm{cm}$ & $1.310^{15} \mathrm{~cm}^{-3}$ & $(100)$ \\
\hline
\end{tabular}

Table 2- Semiconducting Properties of Silicon Substrates

The silicon samples used in this study were double polished to ensure a uniform and reproducible metal contact could be made to the backside of the silicon. The silicon substrates used in this study underwent a thorough organic cleaning in acetone and methanol followed by a 2 minute immersion in a $1 \%$ solution of hydrofluoric acid and DI water. The purpose of the organic cleaning was to remove any native organic materials like oils that may have accumulated on the surface of the silicon during processing, shipment and storage. The purpose of the hydrofluoric acid bath was to ensure that no native oxide remained on the surface of the wafer 
before the deposition process was begun. During the course of this study it was determined that even a few angstroms of native oxide are enough to impede the function of the semiconductorAluminum Nitride - metal structures. This result will be used as supporting, circumstantial evidence for the central conclusion drawn later in this work.

The MOVPE Aluminum Nitride used in this study was deposited in a horizontal AIXTRON 200/4 RF-S Metal Organic Vapor Phase Epitaxy reactor. Trimethylaluminum and ammonia were used as the $\mathrm{Al}$ and $\mathrm{N}$ precursors with approximate flows of $12 \mathrm{~mol} / \mathrm{min}$ and 1.5 slm, respectively. Hydrogen was used as the carrier gas and growth was conducted at a reactor pressure of 50 mbar and an approximate temperature of $1100{ }^{\circ} \mathrm{C}$. The deposition of Aluminum Nitride by MOVPE is inherently a very complex process and therefore falls outside the scope of this work. Suffice it to say that the samples used in this study were processed by Kalyan Kasarla and Lee Ellen Rodak and the specific process parameters can be found in their relevant work. [11]

As previously mentioned the specific process parameters are outlined in Appendix A. Several other process considerations that impact the deposition of Aluminum Nitride shall be outlined here. The first process consideration is the vacuum chamber pressure. It has been generally found that film quality is inversely proportional to the chamber pressure. Hence, lower chamber pressures produce higher quality films. Intuitively one would assume that chamber pressure should be reduced to very near zero. However, at very low chamber pressures it becomes increasingly hard to sustain plasma in the reaction chamber. Due to the challenges inherent in depositing Aluminum Nitride at very low pressures a chamber pressure of 30mTorr was used for the samples in this study even though successful deposition of Aluminum Nitride was demonstrated by this group at pressures as low as $20 \mathrm{mTorr}$. A second critical process 
consideration is the formation of a native oxide on the sputter target. While the vacuum chamber is exposed to ambient conditions a layer of Aluminum Oxide and possibly even Aluminum Nitride forms on the sputter target. To keep this unwanted layer of Oxide or Nitride from contaminating the sample it is imperative to pre-sputter for 5-10 minutes in a pure Argon atmosphere to ensure that this layer is removed from the target. A third critical process consideration that greatly influences both the film quality and the deposition rate is the power applied to the sputter target. In this study all samples were deposited using 500 watts applied to the target. It was found through iterative studies that higher powers generally lead to lower quality films while increasing the deposition rate and lower powers increase the film quality to a point while leading to decreased deposition rates. The result of the iterative studies showed that 500 watts is a near optimum power to deposit polycrystalline Aluminum Nitride. One final process consideration that can easily lead to films with little or no piezoelectric response is the leak rate of the vacuum chamber. It is imperative that the chamber be leak free as oxygen contents as low as $2 \%$ in the films will cause a serious degradation of the piezoelectric response. Because of this the chamber must be fully sealed and pumped to a base pressure of at least $5 \times 10 \mathrm{e}-6$ Torr. At pressures this high out gassing of the chamber is not a significant issue. However, any opportunistic leaks will present themselves at this pressure making periodic helium leak tests a necessity. 


\subsection{Fabrication of MIS Devices}

The fundamental structure used in this study to determine the piezoelectric coefficient of Aluminum Nitride is the Metal Insulator Semiconductor device. It can be argued that the nomenclature used here is fundamentally flawed because Aluminum Nitride is not an insulator but rather a wide bandgap semiconductor with $\mathrm{Eg}=6.22 \mathrm{eV}$. However, in an effort to preserve the nomenclature used in most literature the term Metal Insulator Semiconductor or MIS is used here as opposed to Metal Semiconductor Semiconductor or MSS which may fundamentally be more appropriate. The fundamental operation and implementation of MIS devices has been outlined previously in this work and this section is devoted to the actual implementation of Aluminum Nitride MIS devices as they were used in this work.

The first step in fabricating the Aluminum Nitride MIS devices used in this study was to obviously deposit, either by MOVPE or sputtering, an Aluminum Nitride thin film on a silicon substrate. After the film has been deposited it is possible to apply both topside and backside electrical contacts to the film using standard photolithographic techniques. Solid, circular contacts from a circular TLM pattern were used as illustrated in Figure 9.
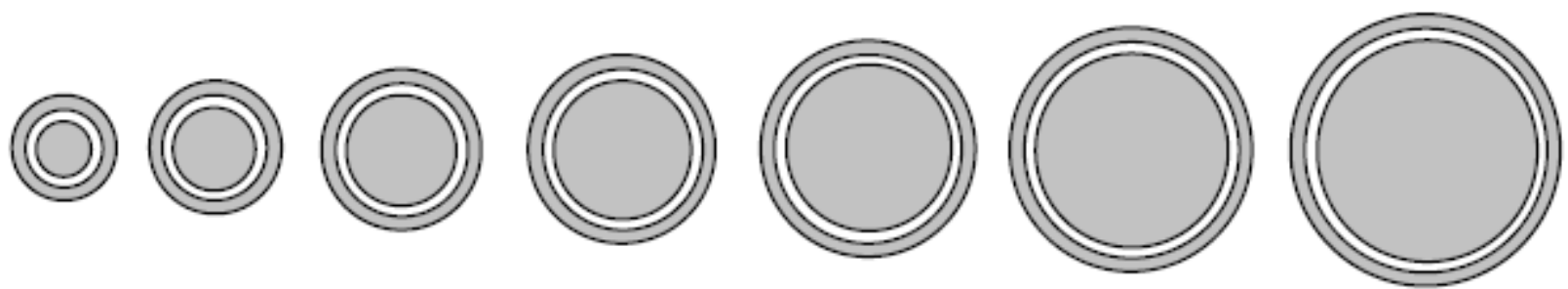

Figure 9 - Circular TLM Pattern

It should be noted that only the inner solid contact was used in this study. The TLM mask used has both rings, circles and ring-circle structures. Only the solid circles were used. To successfully apply a metallic topside contact to the Aluminum Nitride a standard cleaning 
process using acetone and methanol was performed to remove any grease or other organic contaminants that may have survived as remnants of the sputtering process. Next, a dehydration bake is performed for 10 minutes at $100^{\circ} \mathrm{C}$ to ensure the surface of the film is free of any water. After the dehydration a 1.4 micron thick film of photoresist was applied to the samples using a standard centrifugal spin coater. After spin coating, a negative photolithographic process is carried out to the sample to open windows in the photoresist. It should be noted that the developer solution used to expose the underlying Aluminum Nitride can actually etch the Aluminum Nitride film causing the sample to be ruined. This is evidenced when the color of the underlying Aluminum Nitride becomes non uniform and is an indication of overdevelopment. After the windows to the Aluminum Nitride have been opened by the developing process, the topside metallic contacts may be deposited by a standard sputter deposition. Generally the thickness of the metal contacts used in this study was around $250 \mathrm{~nm}$. After the sputter deposition of the topside contact the solid aluminum backside contact was applied using the same sputter deposition process. After the application of the backside contact the samples were subjected to a final acetone bath to facilitate the liftoff process. The acetone dissolves the underlying photoresist and leaves behind only the metallic circular topside contacts. This is the last step in the fabrication of the MIS structure. Figure 10 shows the final contact. 


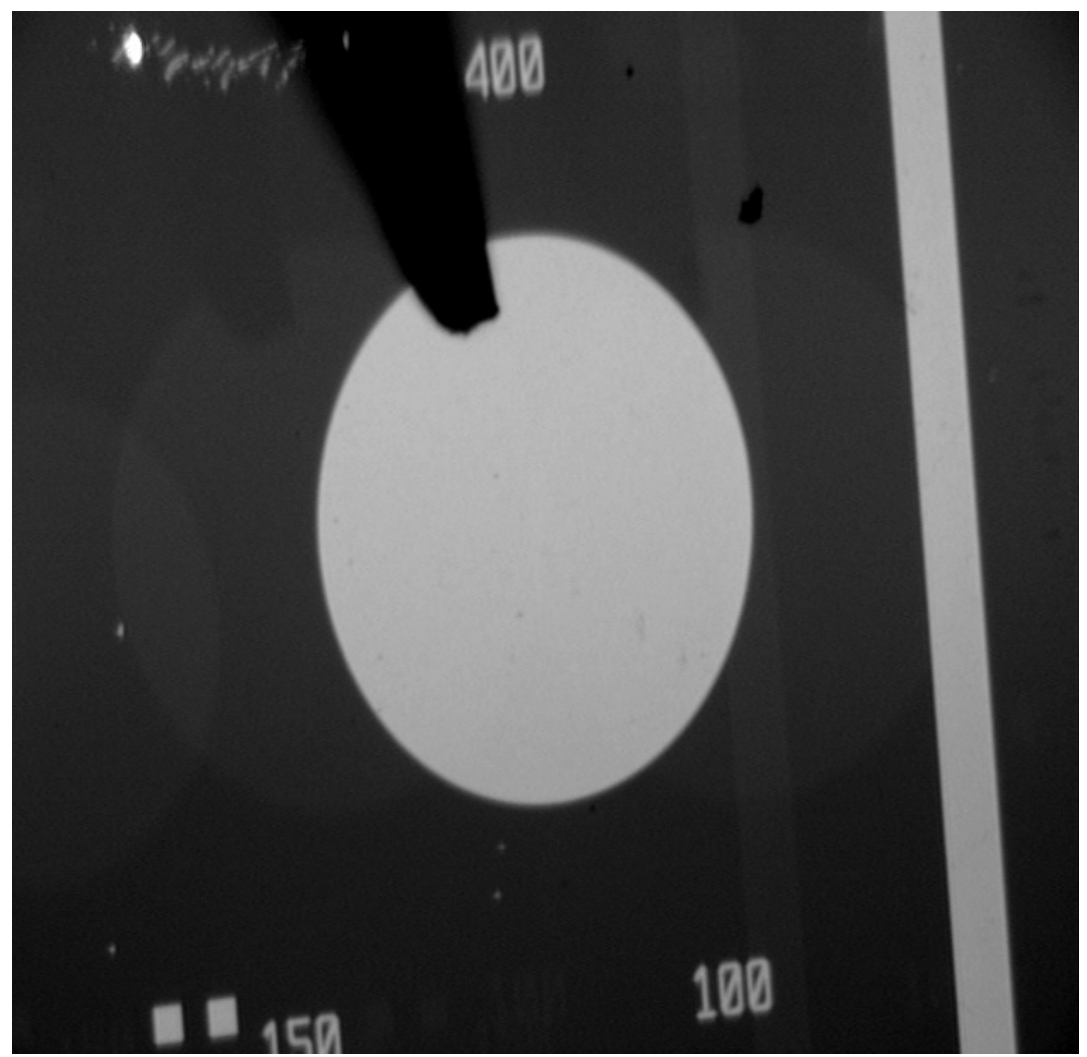

Figure 10 - Metal contact on Aluminum Nitride.

\subsection{Determining the Piezoelectric Coefficient of Aluminum Nitride with LDV}

After fabrication of the MIS device the piezoelectric coefficient of the Aluminum Nitride thin film was determined using a Laser Doppler Vibrometer (LDV). The LDV is a complex instrument that relies on the Doppler shift between two beams of laser light, namely a measurement beam and a reference beam, to make a very accurate determination of the frequency that a solid object is vibrating at. By processing the vibration measurement it is possible to determine the magnitude of the vibration in the frequency domain. The specific protocol used to operate the LDV is outlined in Appendix C. In this work a square wave was applied between the topside and backside contacts to induce an oscillating electric field across the Aluminum Nitride. The magnitude of the response observed by the LDV is directly 
proportional the magnitude of the voltage applied to the sample. Measurements were taken at voltages ranging from 5 to 40 volts peak to peak amplitude and the piezoelectric coefficient was derived from this experimental data.

Several considerations must be made when taking data with the LDV. First, the position of both the reference beam and the measurement beam is critical to obtaining an accurate measurement. The fringing electric field lines induce a piezoelectric response in the Aluminum Nitride some distance from the contact pad. Because of this it is imperative that the reference beam be kept as far as possible away from the measurement beam. Figure 11 illustrates the fringing fields associated with a 40V DC signal across the MIS structure. Figure 11 was produced using Maxwell 2D® field simulator.

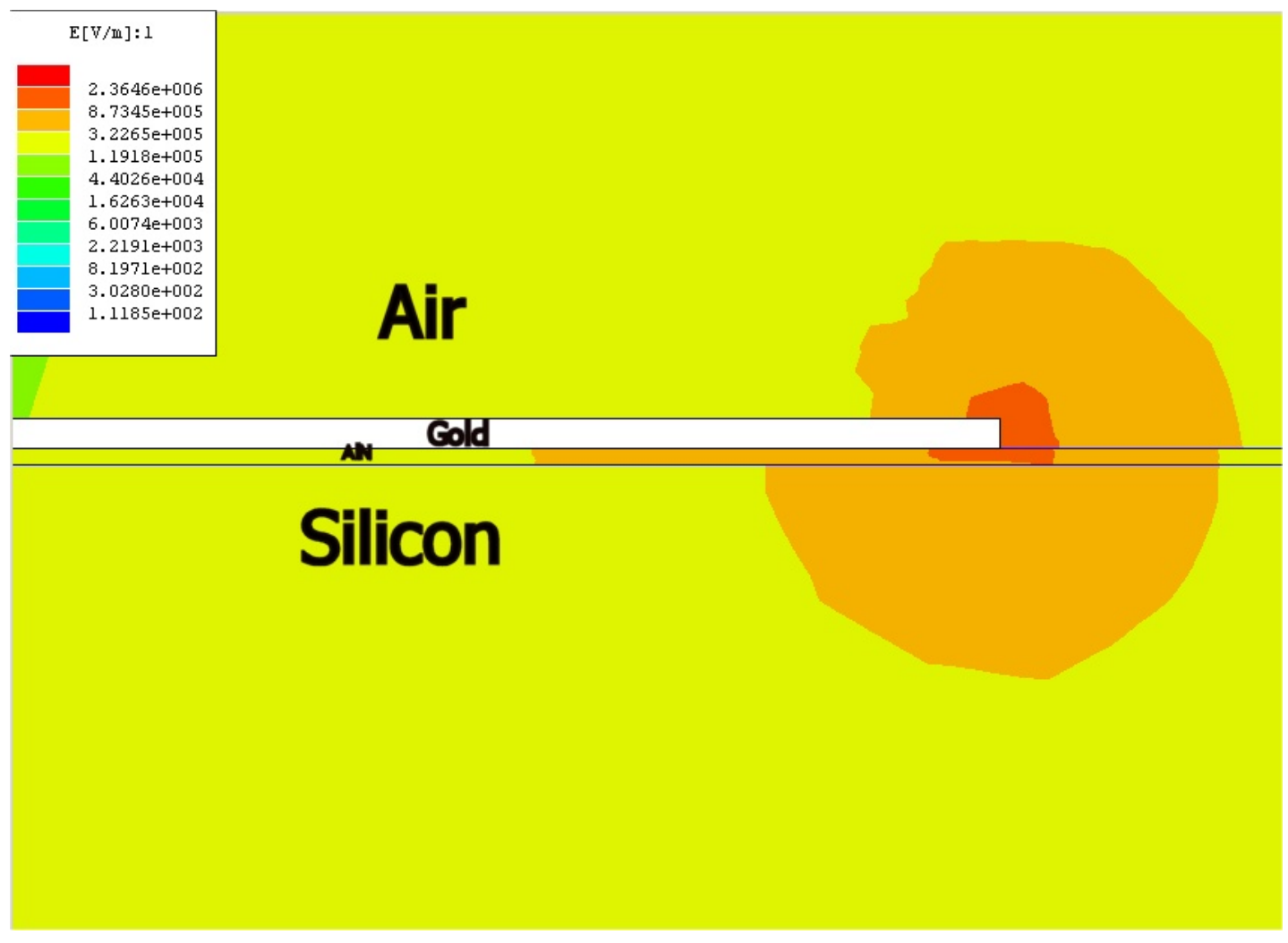

Figure 11 - Fringing Fields on Aluminum Nitride MIS Structure 
As is evidenced in Figure 11 it is possible to observe a falsely high piezoelectric response on the edges of the contact and even on the surrounding Aluminum Nitride film. Because of this effect it is very important to measure the piezoelectric response in or as close to the center of the contact as possible. Further electric field modeling reveals that it is important to measure the piezoelectric response using a sufficiently large contact area to ensure that the electric field vectors are perpendicular to the Aluminum Nitride thin film. Figure 12 and Figure 13 show the effect of varied contact size on the orientation of the electric field lines. The data in Figure 12 and Figure 13 was obtained using Maxwell 3D® field simulator.

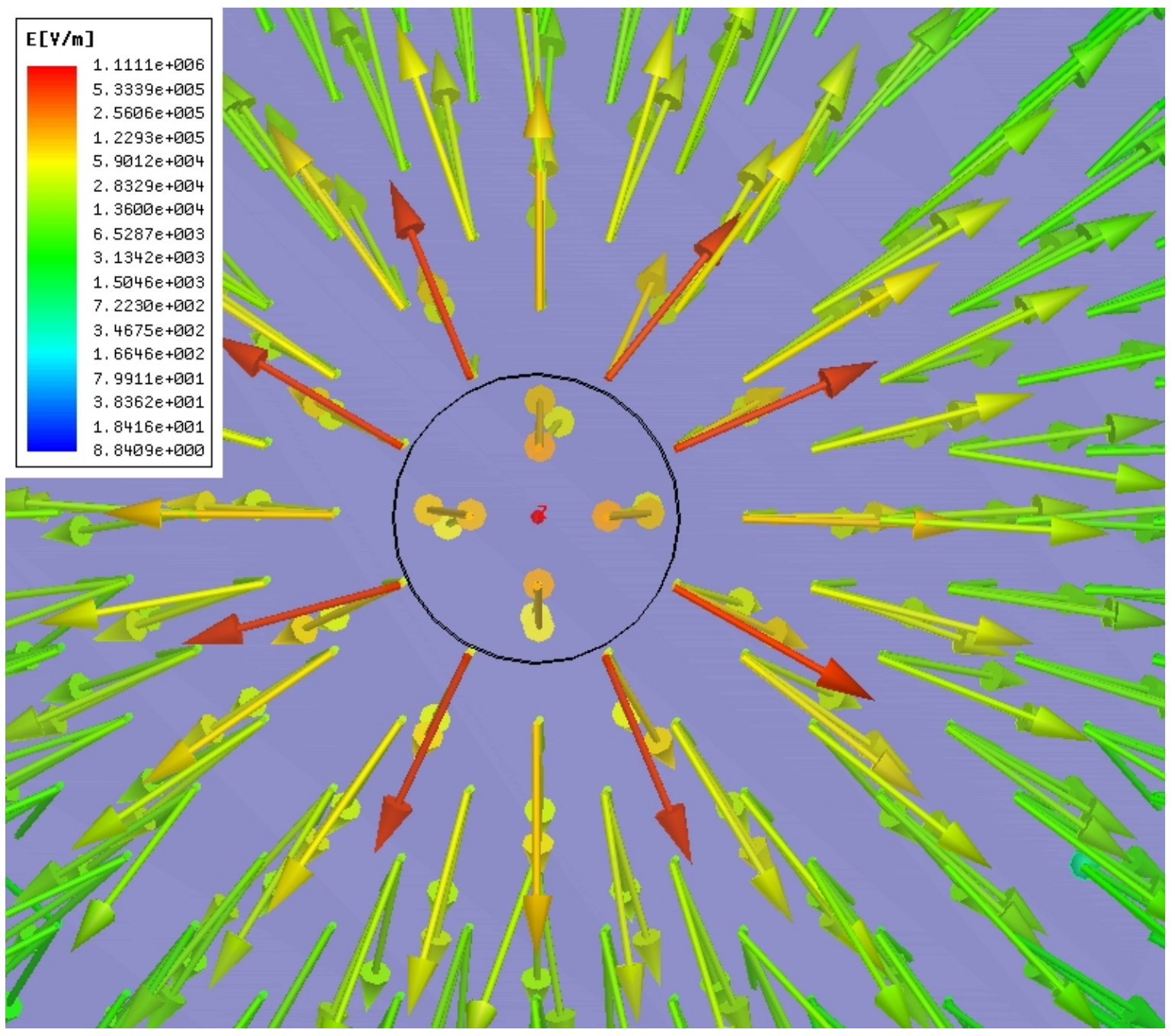

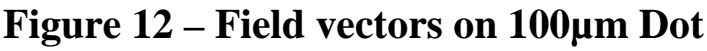




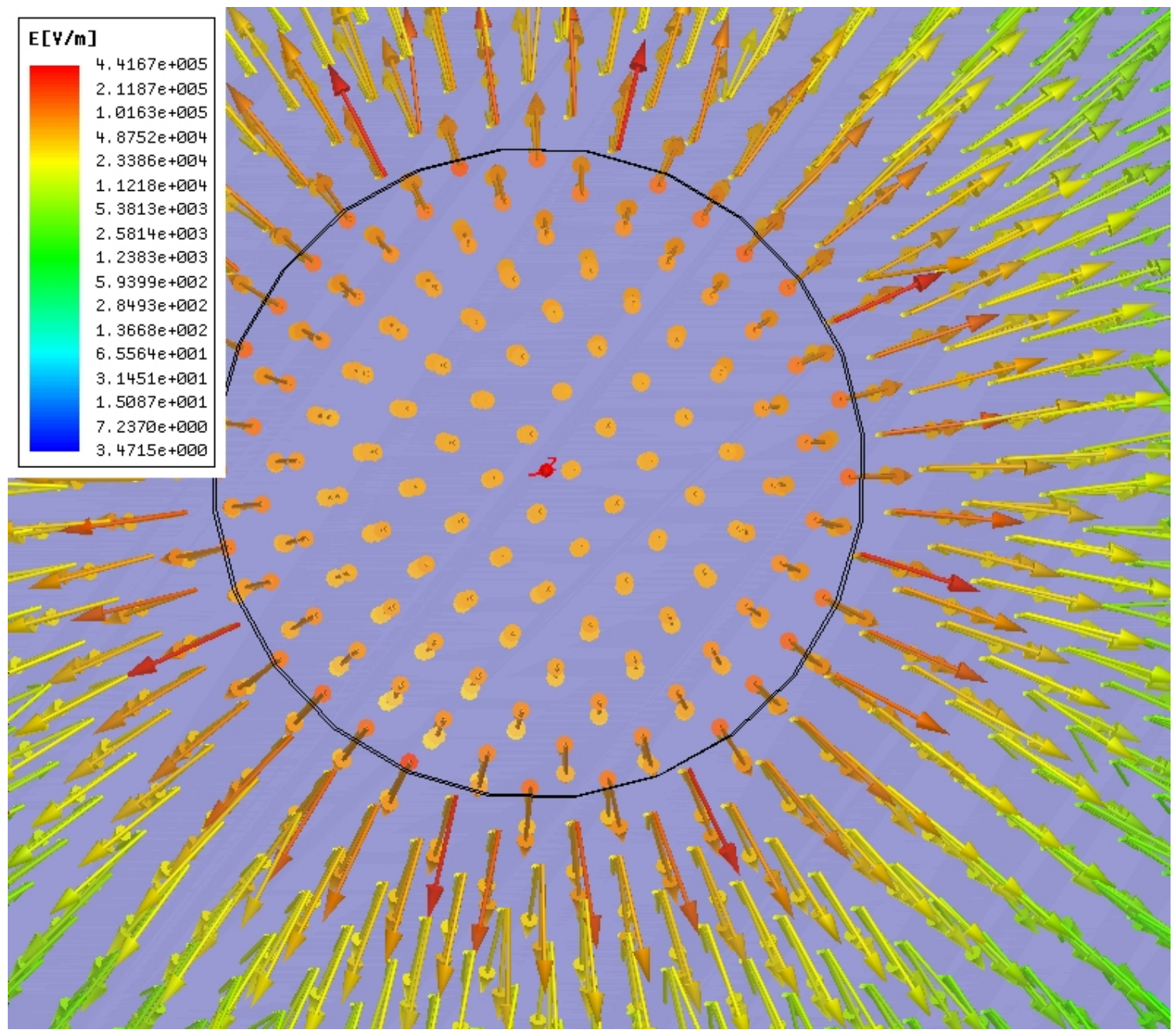

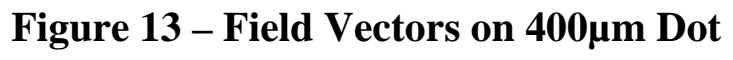

In Figure 12 it is evident that each vector inside the dot has a significant off axis component that effectively reduces the piezoelectric response of the underlying Aluminum Nitride film. However, in Figure 13 the field lines near the center of the dot have almost no off axis component and therefore will give a greater and more accurate piezoelectric response. It should be noted that the field vectors in reality are infinitesimally small meaning that there is a region on the 100um dot where the field vectors have very little off axis component. However, this area is so small and so near the geometric center of the dot that it is impractical to actually try to hit 
an area this small with the laser beam. Therefore, to reduce variability and increase accuracy it is advantageous to use a dot with a larger diameter.

\subsection{Analysis}

The result of any experimental endeavor is contingent upon not only the quality of the empirical data but also upon the analysis techniques used to interpret the data. In this study every possible measure was taken to ensure both the quality of the data and the soundness of the analysis techniques employed to interpret the data. The previous section outlined how the raw data was gathered. After this data was gathered for a particular sample a rigorous method of statistical analysis was employed. Figure 14 is a representative graph of displacement vs. voltage for an Aluminum Nitride sample. 


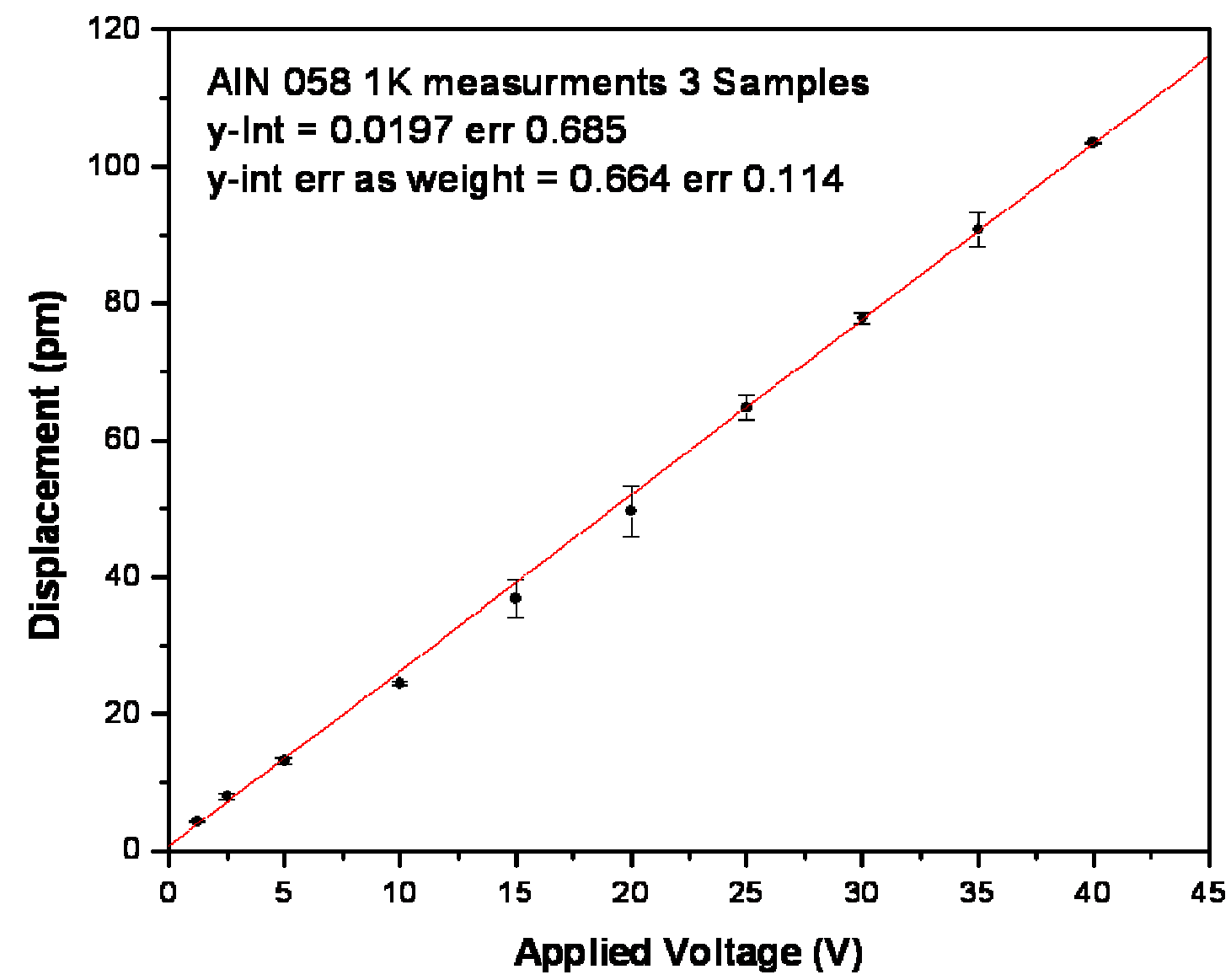

Figure 14 - Typical graph of displacement vs. voltage

The process involved in obtaining the data illustrated in Figure 13 is as follows:

1. A voltage between 5 and $40 \mathrm{~V}$ peak to peak was selected at random. Note that for some high resolution measurements voltages lower than 5 volts were used as is evidenced in Figure 14.

2. The average of either 100 (normal resolution) or 1000 (high resolution) measurements were taken for this specific voltage.

3. Step 2 was repeated 3 times and the average and standard deviation was computed to determine error bars for the measurement. 
4. A standard least squares linear regression was used to compute the line of best fit through the data points.

By using the aforementioned method it is possible to obtain measurements with minimum error. One significant drawback to the LDV software is the fact that it does not compute the standard deviation for the data. Therefore, with a sample size of 100 or 1000 it is impossible to determine the width of the distribution. This inherent inaccuracy is mitigated by taking 3 sets of data for the same point and computing the average and standard deviation. As evidenced in Figure 14 the error is generally very small. It should also be noted that the measurement voltages are chosen at random to ensure that previous measurements have no effect on the subsequent measurements.

\section{EXPERIMENTAL RESULTS}

\subsection{Metals Used}

The experimental setup described in the previous sections was initially devised by the author after doing a comprehensive literature search and determining what assets were available at West Virginia University to measure the piezoelectric coefficient of Aluminum Nitride. The first sample of DC reactive sputtered Aluminum Nitride that was characterized exhibited a larger than expected piezoelectric coefficient. The author chose gold as the topside contact because gold generally wets the surface of III-Nitrides well. The gold contacts also serve as a highly reflective surface for LDV measurements. Initial measurements of the piezoelectric response of sputtered Aluminum Nitride indicated that the $d_{33}$ was greater than $6 \mathrm{pm} / \mathrm{V}$. The theoretical limit of the piezoelectric coefficient of Aluminum Nitride had been shown to be on the order of $4 \mathrm{pm} / \mathrm{V}$. However, other groups had reported measuring the piezoelectric coefficient of Aluminum Nitrite 
at greater than $5 \mathrm{pm} / \mathrm{V}$. These groups attributed this increased $d_{33}$ to the superior quality of the films. Understanding that a fundamental limit cannot be surpassed simply by having a superior quality film the decision was made to investigate the effect on $d_{33}$ of varying the topside contact metal. A hypothesis was generated that the apparent piezoelectric response of the material may be linked to the work function of the metal used as the topside contact and thus the band alignment between the metal and semiconductor. To test this hypothesis 4 readily available metals covering a range of work function from $4.28 \mathrm{eV}$ to $5.65 \mathrm{eV}$ were chosen to conduct the test. The metals used in this study were Aluminum (4.28eV), Titanium (4.32eV), Gold $(5.1 \mathrm{eV})$ and Platinum $(5.65 \mathrm{eV})$. The value of the work function presented here represents the value quoted in the Handbook of Chemistry. Realistically the work function value is a range that depends on several factors. However, for the purposes of this study the difference in relative work function is much more important in establishing trends than the actual work function value. Therefore, an in depth analysis of the techniques used by various groups to measure the metal work function is omitted. All metals used in this study were sputter deposited using Argon gas.

\subsection{Metal Semiconductor Band Alignment}

Metal - semiconductor junctions are very well understood and have been studied for many years and is taught in every introductory device electronics textbook. Despite our excellent understanding of these junctions and our successful application of the idealized theory to practical devices there exist regimes that are not very well understood. One such regime is the contact between a metal and a wide bandgap semiconductor like Aluminum Nitride. It was impossible to determine the Fermi level of the Aluminum Nitride samples used in this study and to assume they are intrinsic with $\mathrm{E}_{\mathrm{f}}=1 / 2 \mathrm{E}_{\mathrm{g}}$ is a very naïve assumption at best. A very small concentration of impurities can lead to a significant shift in the Fermi level. It is almost a 
certainty that the films used in this study have some level of impurity that shifts the Fermi level and makes the films either lightly n-type or lightly p-type. Hall effect measurements are ineffective in determining the concentration of impurities in Aluminum Nitride films simply because no metal exists that makes an ohmic contact to Aluminum Nitride. To begin to get an understanding of the band structure of the MIS structures used in this study Adept, a freely available tool from Purdue University, was used to compute band diagrams for several structures. Despite the previous notion that the Fermi level of AlN is not $1 / 2 \mathrm{E}_{\mathrm{g}}$ the band alignment in the nominal case of $E_{\mathrm{f}}=1 / 2 \mathrm{E}_{\mathrm{g}}$ is an excellent place to begin to understand the band alignment and the associated electric fields.

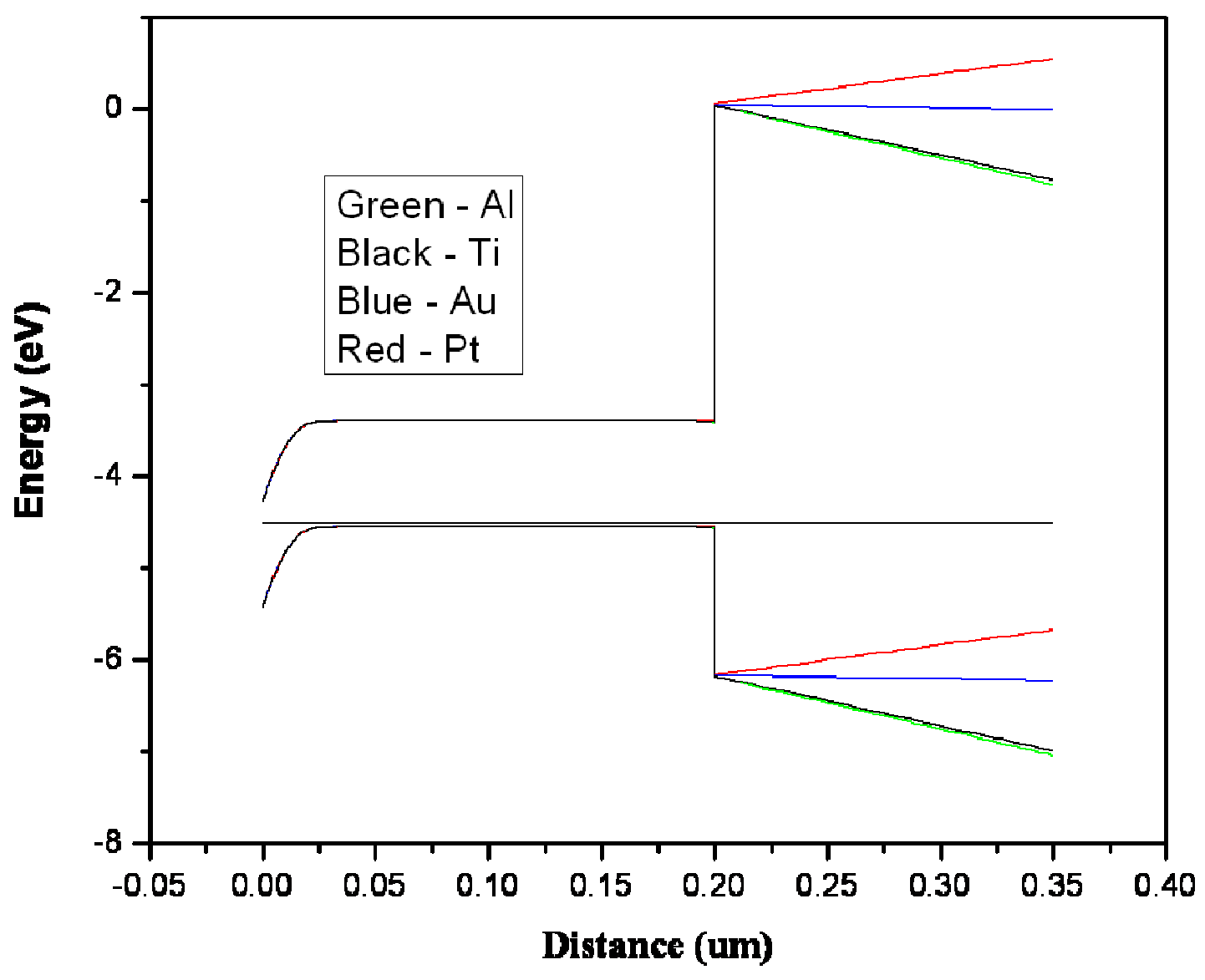


Figure 15 - Band alignment between p-silicon and undoped Aluminum Nitride

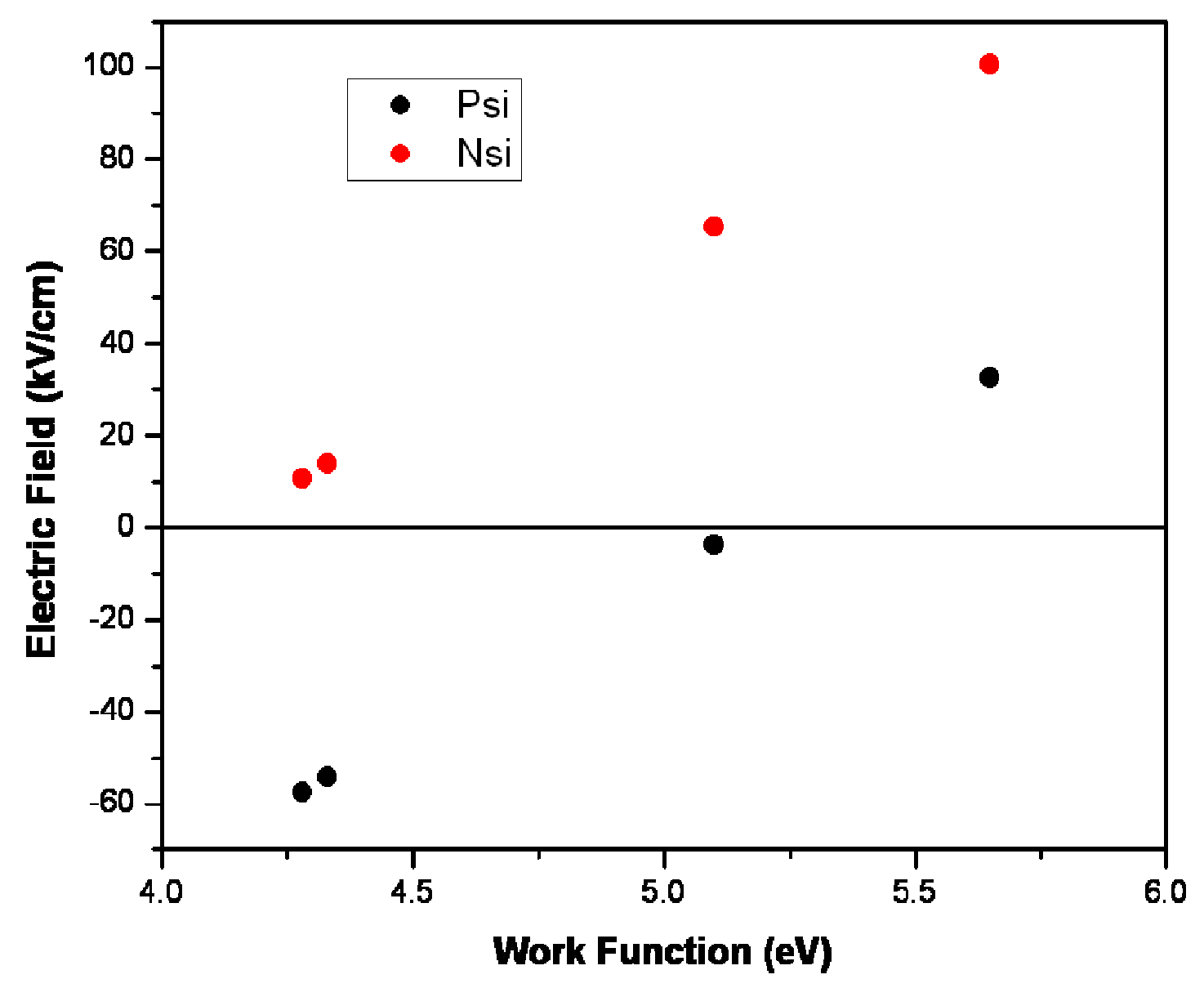

Figure 16 - Maximum Electric Field Across Intrinsic Aluminum Nitride

From Figure 16 which is simply a derivative of Figure 15 it is evident that very significant electric fields do exist in Aluminum Nitride MIS structures. These electric fields undoubtedly have an effect on the initial condition of the Aluminum Nitride crystal and presumably affect the observed piezoelectric response. Further Adept simulations of p-type and n-type Aluminum Nitride were performed with similar results. 


\subsection{Piezoelectric Coefficient Work Function Dependence}

During the course of this study it was found that the apparent piezoelectric coefficient of Aluminum Nitride depends heavily on the metal used as the topside contact. This effect is present for samples deposited by both DC reactive sputtering and MOVPE. Figure 17 shows the work function dependence that is central to this work. All samples in Figure 17 were deposited on a p-silicon substrate.

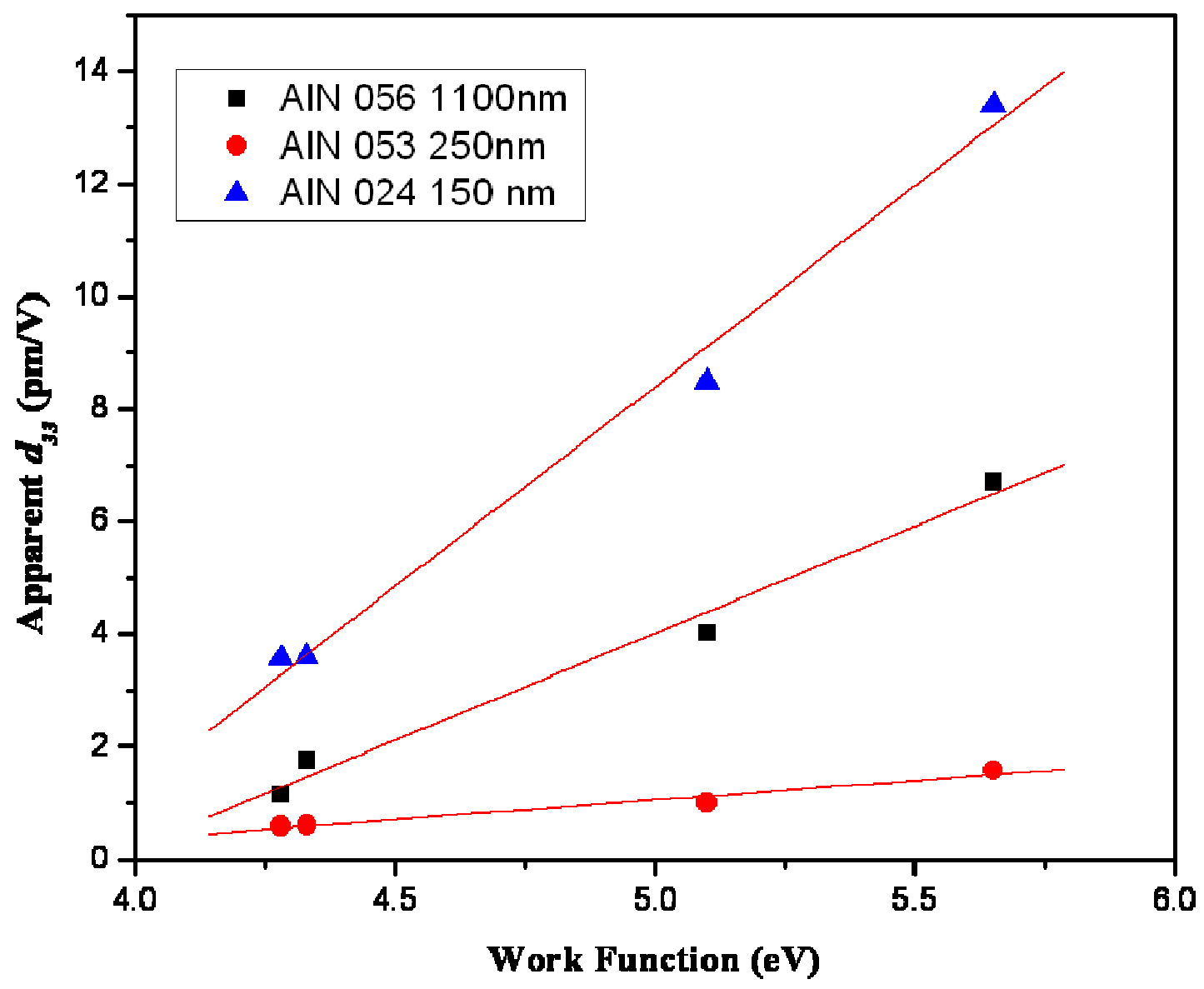

Figure 17 - Piezoelectric Coefficient Dependence on Work Function

As noted in previous sections the metals used in Figure 17 are Aluminum $(4.28 \mathrm{eV})$, Titanium (4.33eV), Gold $(5.1 \mathrm{eV})$ and Platinum $(5.65 \mathrm{eV})$. The data presented in Figure 17 clearly shows a 
linear trend between work function and piezoelectric response. The data represented in Figure 17 illustrates 12 separate samples and over 90,000 individual measurements. Sample 024 was an MOVPE deposited sample with thickness of $150 \mathrm{~nm}$. The response of this sample is much greater than the response of the two DC reactive sputtered samples. The greater response can be attributed to the inherently higher quality films deposited by MOVPE. A qualitative comparison of Figure 17 and Figure 16 show that the piezoelectric response of Aluminum Nitride appears to be closely linked to the electric field across the Aluminum Nitride. The magnitude of the electric field is of course increased as the meal work function is increased thus increasing the apparent piezoelectric response.

The experimental method employed here was studied closely for flaws and all measures were taken to ensure that the results were not influenced by experimental artifacts. All individuals involved in this study agree that the methodology is sound and the results reflect an actual physical process and not an experimental artifact. The results presented here represent the first time that the apparent piezoelectric response of a material has been linked to the metal used as a contact.

\subsection{Perturbation of Band Structure}

To ensure that the data gathered in this study was not flawed it became apparent that the piezoelectric coefficient must be measured under perturbed conditions. This means that the band structure must be altered in a predictable, reproducible fashion that must not interfere with the LDV measurement. 


\subsubsection{Bias}

The most obvious method of perturbing the band structure of the Aluminum Nitride MIS structures is to add a DC bias to the square wave signal applied during the measurement. By applying a DC bias the initial electric field across the Aluminum Nitride is increased or decreased depending on the polarity of the bias. The function generator used in this study was capable of applying a DC offset to the square wave so the measurements required no extra experimental setup. The maximum DC offset that the function generator could produce was $+10 \mathrm{~V}$ or $-10 \mathrm{~V}$. A $20 \mathrm{~V}$ p-p square wave was shifted positive and negative and then the magnitude of the square wave was modulated to gather the $d_{33}$ data. Representative results of the DC bias experiment are presented in Figure 18. 


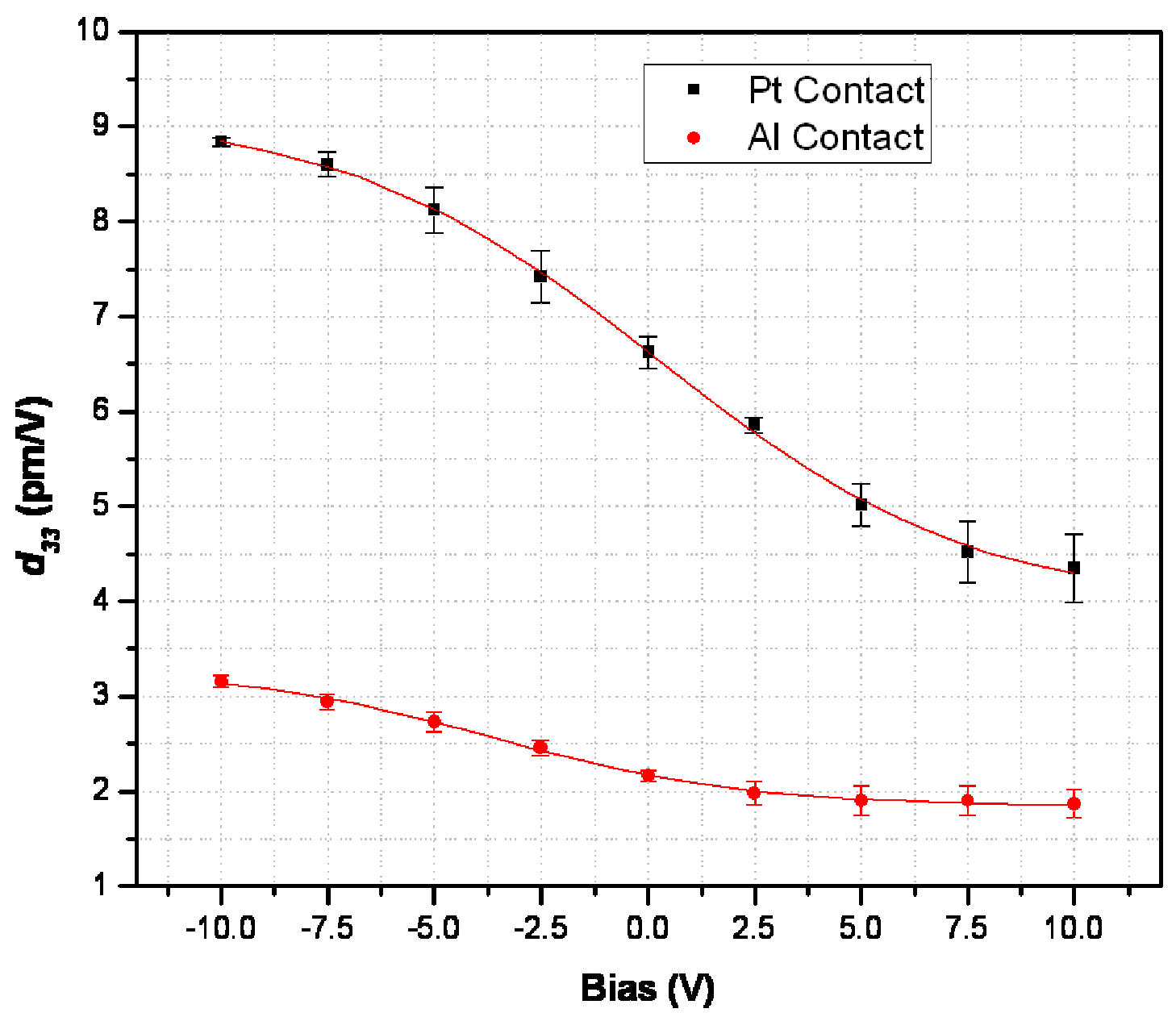

Figure 18 - $d_{33}$ dependence on DC bias of DC reactive sputtered sample.

Figure 18 illustrates that a very pronounced difference in piezoelectric response can be achieved by applying a DC bias to the sample. It is very noteworthy that the Pt contact has a much higher piezoelectric response. However, it should also be noted that the Aluminum sample experienced a $35 \%$ decrease in piezoelectric response from $-10 \mathrm{~V}$ to +10 volts while the Platinum sample experienced a $49 \%$ degradation in piezoelectric response from -10 to +10 volts. It is also noteworthy that the lowest response from the Platinum sample was still almost $50 \%$ higher than 
the highest response of the Aluminum sample. By perturbing the band structure it was possible to confirm that the magnitude of the static field across the Aluminum Nitride plays a large role in determining the apparent piezoelectric response.

To further confirm the band alignment hypothesis another simple method of perturbing the band structure was devised. High intensity white light can be used to optically generate carriers even in a wide bandgap semiconductor. It was also hypothesized that the samples used in this study possess many impurities and mid-bandgap states that allow the generation of a significant number of carriers. To test this hypothesis and to effect another perturbation of the band structure intense white light was focused on the sample while the measurement was being conducted. The results are presented in Figure 19. 


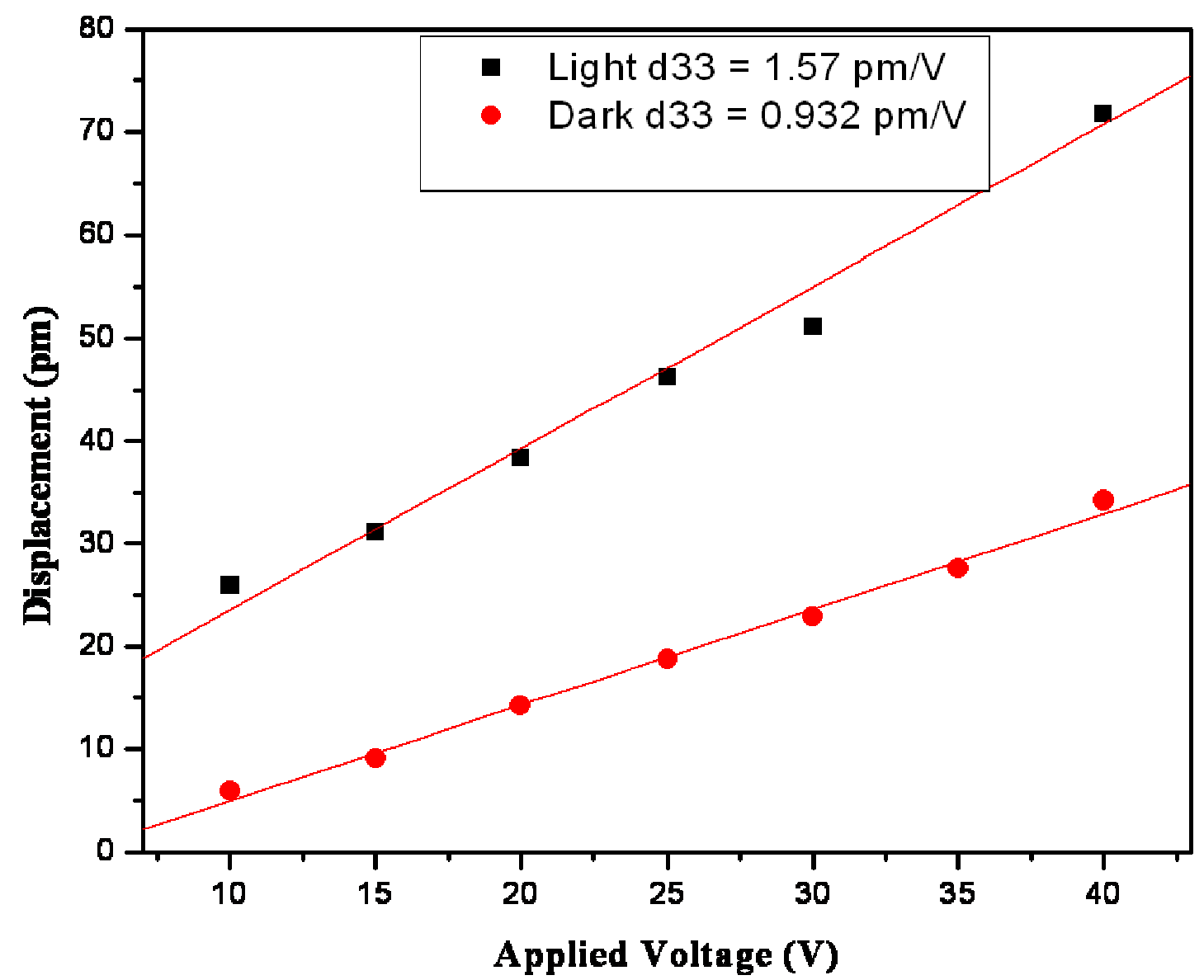

Figure 19 - $d_{33}$ Dependence on Light and Dark Conditions

Figure 19 illustrates that indeed a significant difference in piezoelectric response is observed when the sample is illuminated. As with the DC offset light has a similar but less quantifiable effect on the band structure. To determine if carrier generation at impurities or charge traps in the Aluminum Nitride actually caused a band perturbation that influences the piezoelectric response another simple experiment was devised. A MOVPE deposited film was analyzed under light and dark conditions. It was hypothesized that the MOVPE film, because it is higher quality 
and contains fewer defects, would exhibit much less dependence on the presence of broad spectrum light. Figure 20 illustrates the results of this experiment.

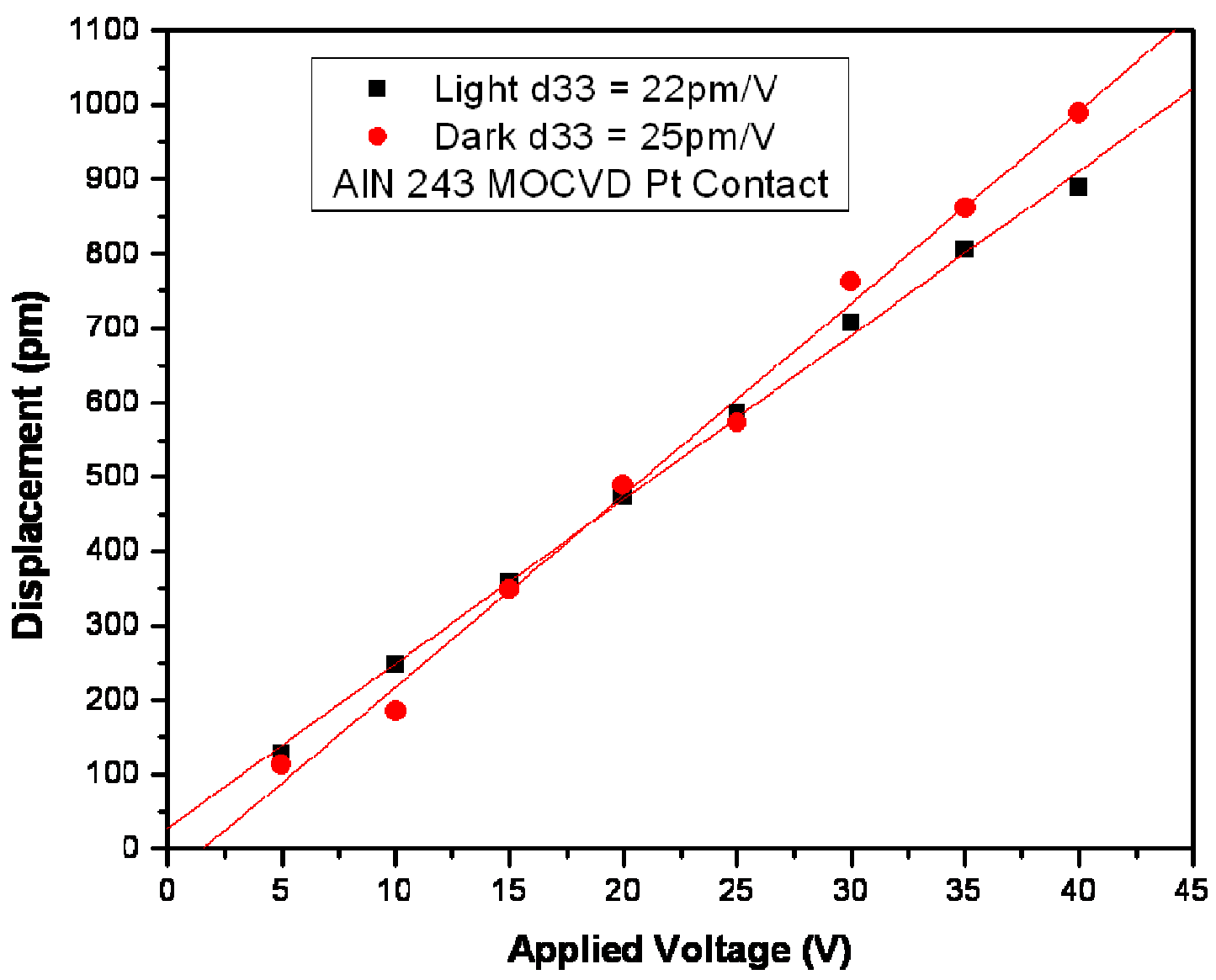

Figure 20 - MOVPE Aluminum Nitride Light and Dark

Figure 20 illustrates that the piezoelectric response of high quality MOVPE Aluminum Nitride is not influenced by the presence of broad spectrum light. This result indicates that carrier generation in DC reactive sputtered Aluminum Nitride occurs primarily at impurities or charge traps. It should be noted that both the MOVPE samples and the DC reactive sputtered samples 
used in this portion of the study were significantly thin to permit light to reach the underlying Silicon. Therefore the effect of optical carrier generation in the Silicon substrate is minimized.

\subsubsection{C-V Hysteresis}

Capacitance as a function of voltage analysis is a widely used semiconductor characterization tool. The capacitance measured from the front to back of a MIS structure in contingent on the DC voltage applied to the structure. This is generally due to the accumulation or depletion of carriers in the semiconductor layer. However, trap states in the insulator layer have been shown to introduce hysteresis into the $\mathrm{CV}$ data. The $\mathrm{CV}$ data used in this study was gathered using an LCR meter and sweeping the DC voltage from positive to negative and back from negative to positive. The resulting data was then plotted to observe any hysteresis present in the structures. The general results of this are evidenced in Figure 21. 


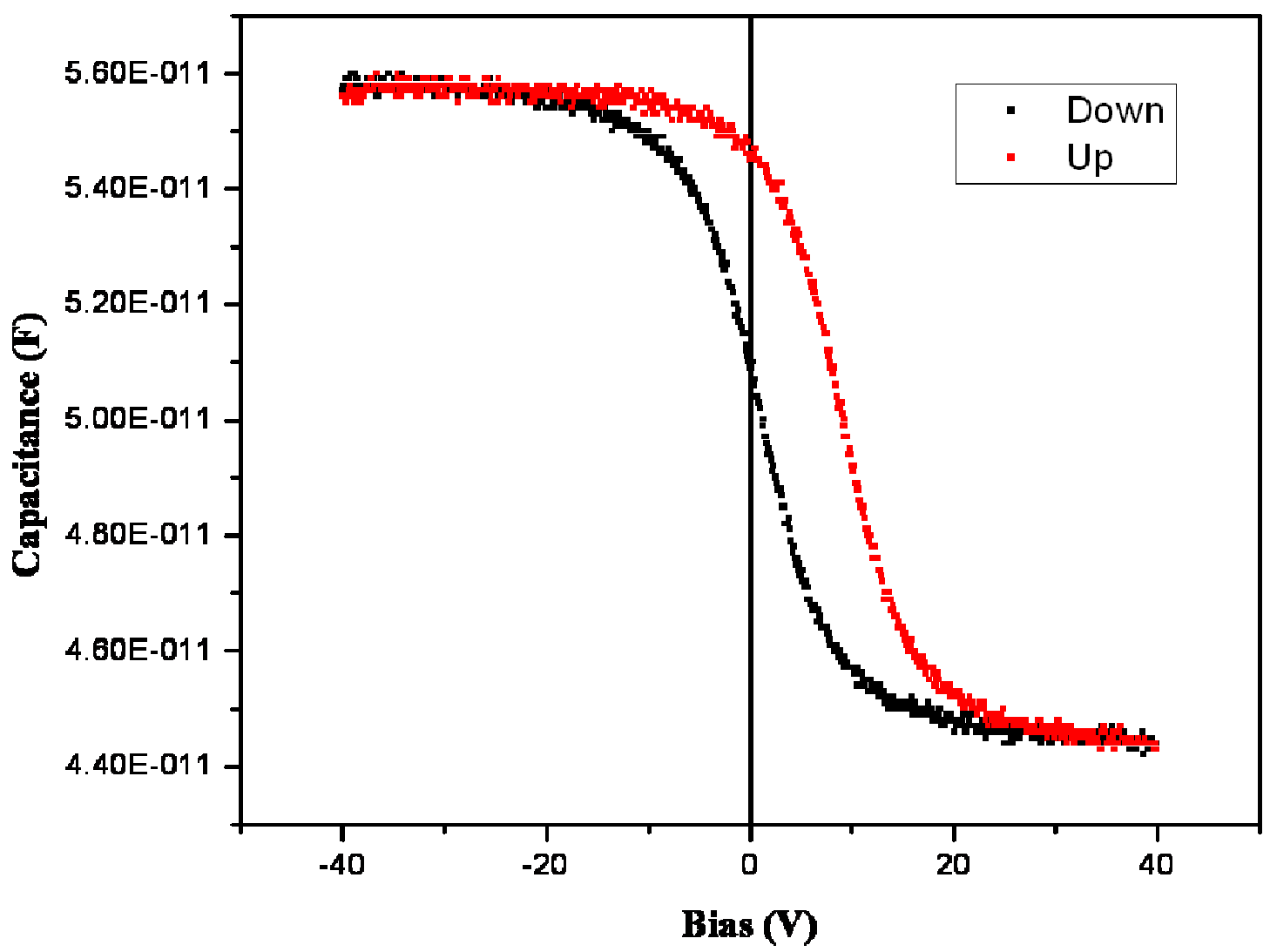

Figure 21 - Aluminum Nitride MIS Hysteresis

Figure 21 outlines the general behavior of the Aluminum Nitride films used in this study. The existence of charge traps in the Aluminum Nitride complicates the understanding of the band alignment and work function dependence on band alignment. Efforts were made to find a relationship between the total trapped charge evidenced by the area between the curves and the work function of the topside contact. These efforts did not produce meaningful or reproducible results indicating that the charge traps are largely a result of film quality and are not affected in any appreciable manner by the composition of the topside contact. It is worth noting that the 
pronounced hysteresis evidence in Figure 20 is not present in MOVPE deposited structures again indicating that fewer impurities and charge traps exist in MOVPE deposited Aluminum Nitride. The results presented here are intended to show that a total understanding of the dependence of $d_{33}$ on work function is not only contingent upon understanding the band structure of the MIS device. The total understanding of this phenomenon is contingent upon understanding many nuanced interactions that influence the response of the device.

\subsubsection{Interpretation of Experimental Results}

The results presented in this work were published in the Journal of Vacuum Science and Technology B in August 2008. The published work focused only on the experimental results and did not attempt an explanation. Throughout the course of this work many experiments have been devised and volumes of supplemental data have been gathered. Despite these efforts a concrete explanation that can be used to both model and predict the behavior of Aluminum Nitride thin films has been illusive. In light of this two promising first order models have been developed that seek to link the piezoelectric response to the electric field induced by the topside metal contacts. These models and their inherent weaknesses are presented.

\section{FIRST ORDER MODEL}

The first order model presented here is based solely on empirical evidence. First consider an Aluminum Nitride thin film with an externally applied electric field. The apparent piezoelectric coefficient of this film is given by the following simple equation.

$$
d_{33}^{A}=\frac{\Delta t}{V_{\text {ext }}}
$$

\section{Equation 5}


Where $d_{33}^{A}$ is the apparent piezoelectric coefficient, $\Delta t$ is the change in thickness and $V_{\text {ext }}$ is the externally applied voltage. It is also possible to define $\Delta t$ in terms of the actual $d_{33}$ and the actual voltages present in the sample. This yields the following equation.

$$
\Delta t=d_{33} V_{t o t}
$$

\section{Equation 6}

Where $V_{\text {tot }}$ is the total voltage across the Aluminum Nitride film defined as the sum of the externally applied voltage and the internal electric fields. Since voltages can be expressed as the product of an electric field over a thickness the following equation can be written.

$$
\Delta t=d_{33} \sum \varepsilon_{x} t_{x}
$$

\section{Equation 7}

Where $\varepsilon_{x} t_{x}$ are the individual internal and external fields. Given this the following equation can be written.

$$
\Delta t=d_{33}\left(\varepsilon_{\text {ext }} t_{\text {film }}+\varepsilon_{\text {int }} t_{\text {int }}\right)
$$

\section{Equation 8}

Where $\varepsilon_{\text {int }}$ is the electric field across the interface and $t_{\text {int }}$ is the thickness of the interface. The terms $\varepsilon_{\text {ext }}$ and $t_{\text {ext }}$ represent the externally applied electric field and total film thickness. Realizing that this product is simply the externally applied voltage the following equation can be written.

$$
\Delta t=d_{33}\left(V_{\text {ext }}+\varepsilon_{\text {int }} t_{\text {int }}\right)
$$

\section{Equation 9}

Replacing $\Delta t$ in Equation 2 with Equation 6 the following equation is generated: 


$$
d_{33}^{A}=\frac{d_{33}\left(V_{\text {ext }}+\varepsilon_{\text {int }} t_{\text {int }}\right)}{V_{\text {ext }}}
$$

\section{Equation 10 - Apparent $d_{33}$ Due to Built In Fields}

Equation 7 shows that the internal electric fields can actually increase or decrease the piezoelectric response of the Aluminum Nitride thin film. The assumption can be made that since the film is completely depleted of carriers the electric field extends across the entire film. Using a 500nm thick film and assuming an electric field of $100 \mathrm{kV} / \mathrm{cm}$ it is possible to see an increase in $d_{33}$ of about 1.125 times nominal. This approach also allows the DC bias data to be explained. By simply adding another term to Equation 7 it is possible to take into account the DC bias dependence. However, this approach does not take into account the non-linearity of the DC bias data and the nearly maximum values used to derive the 1.125 increase factor do not account for the experimental data.

It is very clear from this approach that other unquantified factors play a significant role in this study. The existence of trap states, both surface and mid-bandgap, complicate the study.

\section{CONCLUSIONS AND FUTURE WORK}

The results outlined in this work represent a major step forward in the understanding of piezoelectric semiconducting materials and have directly led to three peer reviewed publications. [20], [11], [21] However, this work is lacking in that it only reports a result and presents a plausible hypothesis supported by circumstantial evidence. More work, both experimental and analytical, is needed to bring a full analytical solution to light. The search for a full analytical solution will undoubtedly uncover other previously unknown phenomenon. It is the authors hope that this work can continue past this preliminary study. 


\section{REFERENCES}

1. Optoelectronic devices based on GaN, AlGaN, InGaN homo-heterojunctions and superlattices. Khan, M. and M, Shur. San Jose : s.n., 1997. Vol. 2397, p. 283.

2. Study of epitaxial lateral overgrowth of GaN for application in the fabrication of optoelectronic devices. Berry Ann, N. J., et al. s.1. : Proceedings of SPIE, 2005. p. 60170D.

3. Muller, Richard S. and Kamins, Theodore I. Device Electronics for Integrated Circuits. s.l. : John Wiley \& Sons, Inc., 2003.

4. Streetman, Ben G and Banerjee, Sanjay. Solid State Electronic Devices. s.l. : Prentice Hall, 2000.

5. Piezoelectric coefficients of aluminum nitride and gallium nitride. Leung, C. M., et al. San Fransisco, CA : Materials Research Society, 1999.

6. A systematic study on group III-nitride thin films with low temperature deposited via MOCVD. Tai-Chang, Chen, et al. 4, s.1. : Optical Materials, 2004, Vol. 26.

7. III-Nitride semiconductor growth by MBE: Recent issues. Morkoc, H. 12, s.1. : Journal of Materials Science: Materials in Electronics, 2001, Vol. 12.

8. Single-phase aluminum nitride films by dc-magnetron sputtering. Morgan, J. S., et al. 11, s.1. : Journal of Materials Research, 1990, Vol. 5.

9. Study of piezoelectric aluminum nitride thin film by RF magnetron sputtering. Wu, Shih-Jeh and Shen, Ting-Wai. Shatin, China: IEEE International Conference on Robotics and Biomimetics, 2005.

10. Characterization of piezoelectric aluminum nitride thin film by RF magnetron sputtering. Wu, Shih-Jeh, Shen, Ting-Wei and Kuo, Chen-Ming. San Antonio, TX : Trends in Materials R and D for Sensor Manufacturing Technologies, 2006. 
11. High temperature annealing studies on the piezoelectric properties of thin aluminum nitride films. Farrell, R., et al. Boston, MS : Microelectromechanical Systems - Materials and Devices Symposium, 2007. pp. 191-5.

12. Polycrystalline grain structure of sputtered aluminum nitride films. Matsuda, Asher T., et al. San Fransisco, CA : Materials Research Society Symposium, 1994.

13. Turlapati, Lavanya. Developing UV Photodetector and Ohmic Contact Techniques on GaN. Morgantown, WV : West Virginia University, 2004.

14. First-principles calculation of the structural, electronic, and vibrational properties of gallium nitride and aluminum nitride. Miwa, K. and Fukumoto, A. 11, s.1. : Physical Review B (Condensed Matter), 1993, Vol. 48.

15. Dielectric breakdown in aluminum nitride. Ruemenapp, T. and Peier, D. London, United Kingdom: Proceedings of the 1999 11th International Symposium on 'High Voltage Engineering, 1999.

16. Electronic and optical properties of rock-salt aluminum nitride obtained from first principles. Zhang, Xinyu, et al. 42, s.l. : Journal of Physics Condensed Matter, 2007, Vol. 19.

17. Optical properties of aluminum nitride thin film. Liu, Wen, et al. 5, s.l. : Journal of the Chinese Ceramic Society, 2007, Vol. 35.

18. Holler, James F., Skoog, Douglas A. and Crouch, Stanley R. Principles of Instrumental Analysis,. s.l. : Cengage Learning, 2007.

19. Piezoelectric coefficient measurment of piezoelectric thin films: an overview. Liu, J. M., et al. s.1. : Materials Chemistry and Physics, 2002, Vol. 75.

20. Effect of contact metals on the piezoelectric properties of aluminum nitride thin films. Harman, J., et al. 4, s.1. : J. Vac. Sci. Technology B, 2008, Vol. 26. 
21. Development of aluminum nitride / platinum stack structures for an enhanced piezoelectric response. Kabulski, Adam, et al. Boston, MS : Materials Research Society, 2006. 


\section{APPENDIX A - DEPOSITION OF ALUMINUM NITRIDE BY REACTIVE DC MAGNETRON SPUTTERING}

The predominant means of depositing the Aluminum Nitride thin films used in this study was DC reactive magnetron sputtering. The films synthesized by DC reactive magnetron sputtering are generally polycrystalline films with a refractive index in the range of $1.85-1.95$. The sputtering system used was a CVC 610 magnetron sputtering system. To deposit Aluminum Nitride it is necessary to simultaneously populate the chamber with both Nitrogen and atomic Aluminum. Because the Nitrogen molecules are very light it is necessary to introduce the inert gas Argon into the chamber to aid in the sputtering process. The only purpose that Argon serves is to accelerate the sputtering of Aluminum atoms from the target. The $200 \mathrm{~mm}$ diameter target is composed of $99.999 \%$ pure Aluminum and is driven with 500 watts of DC power. The system uses a combination of a mechanical roughing pump and a cryogenic pump to achieve a vacuum level of 5e-6 Torr. It is imperative that this base pressure is reached to ensure that all contaminants, especially Oxygen, are removed from the chamber before deposition. Any Oxygen content above approximately $2.5 \%$ will significantly reduce the piezoelectric response of the material. It is also important to perform a pre-sputter on the target to remove any Aluminum Nitride or Aluminum Oxide that has formed on the target in previous depositions or while the chamber is open. The following steps outline the protocol for deposition of Aluminum Nitride. 
1. Vent the chamber with nitrogen.

2. Open the chamber, mount the sample over the large target and close the chamber.

3. Ensure that the shutter is open on position 2 so it can be opened to position 3 for the deposition.

4. Set the timer to the desired deposition time. The deposition rate is approximately $10 \mathrm{~nm} / \mathrm{min}$.

5. Change the power on the magnetron drive to $500 \mathrm{~W}$.

6. Engage the rough valve and turn the mechanical pump on.

7. When the rough pressure reaches 1 turn the rough valve and the mechanical pump off and turn the high vacuum valve on.

8. When the gas pressure reaches 0.09 turn the ion gauge on and wait until the pressure in the chamber reaches 5e-5 Torr. Turn the ion gauge off.

9. When the base pressure has been reached set the analog mass flow controllers for Nitrogen and Argon to 27 and 3 SCCM respectively. These are gasses 1 and 3 on the mass flow controller respectively.

10. Flow gasses 1 and 3 into the chamber by turning on the gas 1 and 3 buttons and engage the throttle valve by turning on the throttle valve button.

11. Bring the pressure in the chamber to 30 mTorr. This equates to a readout of 3.00 on the pressure gauge.

12. Engage the continuous sputter mode and turn the magnetron drive on.

13. Pre-sputter for 10 minutes then simultaneously turn the shutter to position 3 and engage the timed sputter. 
14. Once the sputtering process is complete turn off the gasses and turn off the throttle valve and high vacuum. When the high vacuum gate valve has closed vent the chamber and remove the sample.

\section{APPENDIX B - FABRICATION OF METAL INSULATOR SEMICONDUCTOR STRUCTURES}

The Metal Insulator Semiconductor (MIS) structures central to this work were fabricated by using standard Silicon processing technology in the clean room environment at West Virginia University. The process outlined here can be used to deposit any metal on the Aluminum Nitride Film. It is assumed that the most fundamental part of this work, the deposition of an Aluminum Nitride thin film, has all ready been performed.

1. Clean the sample with acetone for 5 minutes.

2. Clean the sample with methanol for 5 minutes.

3. Dry the sample with clean room grade compressed Nitrogen.

4. Bake the sample at $100 \mathrm{C}$ for 10 minutes to remove any water.

5. Spin coat the sample with AZ 5214 photoresist at 4000 RPM for 30 seconds.

6. Soft bake the sample at $90 \mathrm{C}$ for 45 seconds.

7. Expose the sample using the desired mask and calculated exposure time.

8. Hard bake the sample at $120 \mathrm{C}$ for 2 minutes.

9. Flood expose the sample for 82 seconds.

10. Develop the sample in a 1:6 solution of AZ 400K developer to DI water for 20 seconds.

11. Immerse the sample in water to remove any residual developer.

12. Dry the sample with compressed clean room Nitrogen. 
13. Look at the pattern under the optical microscope to determine if it is fully developed.

14. Mount the sample in the sputtering chamber.

15. Sputter the topside contact.

16. Remove the sample and sputter the backside contact.

17. Remove the sample from the chamber and immerse the sample in Acetone to facilitate the liftoff process.

18. If necessary put the sample into the ultrasonic bath to help increase the speed of the liftoff process.

19. After the liftoff process is complete clean the sample with DI water and dry the sample with clean room Nitrogen. 


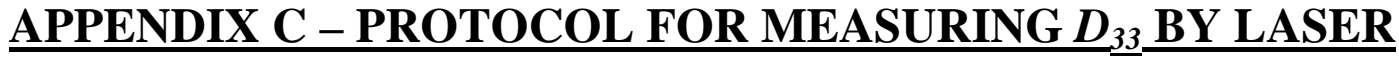 \\ DOPPLER VIBROMETRY}

The piezoelectric measurements used in this study were taken using a dual beam differential Laser Doppler Vibrometer (LDV). To obtain and ensure consistent results it is imperative that an experimental protocol be followed for each measurement. Each sample presents specific challenges with regard to data collection and validation. The following protocol allows consistent measurements and provides enough data to reduce error to the level of background noise.

1. Turn the computer, video camera and laser output unit of the LDV on.

2. Open the controller software on the PC.

3. Allow 20 minutes for the laser to come to a steady state temperature before taking a measurement.

4. Apply a small amount of conductive silver paste to the conductive aluminum plate on the mounting bracket under the microscope.

5. Place the sample contact side up on the conductive silver paste under the microscope.

6. Switch the software to video mode and focus the microscope on the sample.

7. Move the sample using the $\mathrm{x}$ and $\mathrm{y}$ adjustments on the mounting stage until the desired contact is roughly in the middle of the field of view.

8. Turn the laser on and using the laser $\mathrm{x}$ and $\mathrm{y}$ movement controls in the software position the measurement beam on the contact pad.

9. Using the manual controls on the laser scanner head move the measurement beam to the side of the contact pad. 
10. Ensure sufficient signal is present by looking both at the signal bar on the laser output unit and the signal bar displayed on the computer screen.

11. Gently probe the edge of the contact pad with the three axis probe and tungsten probe tip. Once the probe is in contact with the sample do not continue to push the probe onto the contact.

12. In the LDV measurement setup ensure that the software is setup to take 100 single point measurements.

13. Set the output of the function generator to $40 \mathrm{~V} \mathrm{p}-\mathrm{p}$ at $3.5 \mathrm{kHz}$.

14. Ensure the connections between the function generator and the sample (topside and backside) are workable.

15. Ensure that the bandpass filter applied in the LDV software measurement setup is set for a bottom cutoff frequency of less than $1 \mathrm{kHz}$ and the top cutoff frequency is set greater than $5 \mathrm{kHz}$. This ensures that no attenuation at the measurement frequency of $3.5 \mathrm{kHz}$ will occur.

16. Turn on the output of the function generator.

17. Start the measurement on the LDV and allow it to average 100 samples.

18. Repeat this measurement a minimum of three times to ensure the accuracy of the data.

19. Once the data has been validated for a single voltage record the displacement as a function of voltage by varying the voltage randomly and measuring the average displacement for 100 samples.

20. Once a minimum of 8 measurements of 100 samples each have been taken plot the data and perform a linear regression. The slope of the regression line provides the $d_{33}$ of the sample. 
Notes: It is always a good idea to make sure the signal to noise ratio is greater than 5 and preferably 10 . The higher the signal to noise ratio the more accurate the measurement will be. After much study it was found that 100 measurements averaged are functionally as accurate as 1000 or 10,000 . 100 measurements take approximately 1 minute to take while 10,000 take over 1.5 hours. It was noted that not all samples (in fact very few samples) had a regression line that went through the origin. Many samples had intercepts that were significantly greater than the background noise indicating some other source of experimental error or an unknown effect in the film. Despite much study it was not possible to determine the origin of this discrepancy. Future work should disregard this non-zero intercept unless it is an order of magnitude or more greater than the background noise. 


\section{APPENDIX D - PROTOCOL FOR DETERMINING DC BIAS DEPENDENCE OF $\underline{D}_{33}$}

1. Follow the setup procedures for the LDV outlined in Appendix C.

2. Set the function generator to DC offset mode.

3. Apply a DC offset of between -10 and 10 volts to the sample.

4. Apply a $3.5 \mathrm{kHz}$ square wave to the sample of amplitude between 0 and 20 volts.

5. Measure the displacement of the sample following the procedures outlined in Appendix C.

6. Record the data.

7. Change the amplitude of the square wave within the 0 to 20 volt range and repeat the measurement at least 4 times.

8. Calculate the piezoelectric coefficient for that particular offset using standard linear regression.

9. Change the DC offset and repeat steps 5 through 8 . 